\title{
Synthesis of Substituted P-Stereogenic Vinylphosphine Oxides by Olefin Cross-Metathesis
}

\author{
Supplementary Information \\ Oleg M. Demchuk, ${ }^{a}$ K. Michał Pietrusiewicz,
and Karol Grela ${ }^{\mathrm{a} *}$ Anna Michrowska ${ }^{\mathrm{a}}$ \\ ${ }^{a}$ Institute of Organic Chemistry, Polish Academy of Sciences \\ Kasprzaka 44/52, 01-224 Warsaw, Poland \\ b Department of Organic Chemistry, Maria Curie-Skłodowska University \\ Gliniana 33, 20-614 Lublin, Poland \\ kmp@icho.edu.pl,grela@icho.edu.pl
}

\section{General}

Unless otherwise noted, all reactions were carried out under Ar in pre-dried glassware using Schlenk techniques. The solvents were dried by distillation over the following drying agents and were transferred under argon: THF (K/benzophenone), toluene (Na), $n$-pentane, $n$-hexane, $\mathrm{CH}_{2} \mathrm{Cl}_{2}$ $\left(\mathrm{CaH}_{2}\right), \mathrm{Et}_{2} \mathrm{O}\left(\mathrm{LiAlH}_{4}\right)$. Flash column chromatography: Merck silica gel 60 (230-400 mesh). NMR: Spectra were recorded on Bruker AVANCE 500, Varian Gemini 200 and 400 spectrometers in $\mathrm{CDCl}_{3}$; chemical shifts $(\delta)$ are given in ppm relative to TMS, coupling constants $(\mathrm{J})$ in $\mathrm{Hz}$. IR: Perkin-Elmer Spectrum 2000 FT-IR, wavenumbers in $\mathrm{cm}^{-1}$. MS (EI, LSIMS): AMD 604 Intectra GmbH. MS (ESI): Mariner Perseptive Biosystems, Inc. HPLC-MS (API): HP 1100 series with PE SCIEX API 365 turbo-ion spray and Nucleosil 1000-C18 column. Micro-analyses were provided by Institute of Organic Chemistry, PAS, Warsaw. 1c, ${ }^{1} \mathbf{1 d},{ }^{2} \mathbf{2 e},{ }^{3} \mathbf{3 a},{ }^{4} \mathbf{3} \mathbf{b}^{5}$ and $\mathbf{3} \mathbf{c}^{6}$ were prepared according to the literature procedures. All commercially available substrates were used as received.

\section{General procedure for cross-metathesis of vinylphosphine oxides.}

\subsection{Procedure A}

To a mixture of vinylphosphine oxide $3(1.0 \mathrm{mmol})$ and $2(0.5 \mathrm{mmol})$ in $\mathrm{CH}_{2} \mathrm{Cl}_{2}(4 \mathrm{~mL})$ was added a solution of catalyst $1(0.025 \mathrm{mmol}, 5 \mathrm{~mol} \%)$ in $\mathrm{CH}_{2} \mathrm{Cl}_{2}(1 \mathrm{~mL})$. The resulting mixture was stirred at $45{ }^{\circ} \mathrm{C}$ for $16 \mathrm{~h}$. The solvent was removed under reduced pressure. The crude product 4 was purified by flash chromatography (hexane-acetone $4: 1$, then hexane-ethyl acetate-methanol $5: 2: 0.5)$.

1 K. Grela, S. Harutyunyan, A. Michrowska, Angew. Chem. Int. Ed. Engl. 2002, 41, 4038.

2 K. Grela, M. Kim Eur. J. Org. Chem., 2003, 96.

3 N. Moskalev, M. Mąkosza, Tetrahedron Lett. 1999, 40, 5935; (b) N. Moskalev, M. Barbasiewicz, manuscript in preparation.

4 D. J. Collins, Aust. J. Chem. 1974, 27, 2365.

5 (a) K. M. Pietrusiewicz, M. Zablocka, J. Monkiewicz, J. Org. Chem., 1984, 49, 152; (b) ;K. M. Pietrusiewicz, M. Zabłocka, Tetrahedron Lett., 1988, 29, 1991 (c) U. Nagel, C. Roller, Z. Naturforsch. B, 1998, 53, 2211.

6 K. M. Pietrusiewicz, Phosphorus, Sulfur, Silicon, 1996, 109, 573. 
[(1E)-6-tert-butyl(dimethyl)sililoxyhex-1-enyl](diphenyl)phosphine oxide (4a) Colourless oil; 82\% yield; IR $\left(\mathrm{KBr}, \mathrm{cm}^{-1}\right)$ 3059, 2948, 2959, 1617, 1438, 1252, 1189, 1097, 839, 701, 528; ${ }^{31} \mathbf{P}$ NMR $\left(\mathrm{CDCl}_{3}, 202 \mathrm{MHz}\right): \delta=24.8 ;{ }^{1} \mathbf{H ~ N M R}\left(\mathrm{CDCl}_{3}, 500 \mathrm{MHz}\right): \delta=7.71-7.64(\mathrm{~m}, 4 \mathrm{H})$, $7.52-7.40(\mathrm{~m}, 6 \mathrm{H}), 6.71(\mathrm{ddt}, J=19.5,17.0,6.5 \mathrm{~Hz}, 1 \mathrm{H}), 6.22$ (ddt, $J=24.5,17.0,1.5 \mathrm{~Hz}$, $1 \mathrm{H}), 3.61-3.57(\mathrm{~m}, 2 \mathrm{H}), 2.34-2.2 .26(\mathrm{~m}, 2 \mathrm{H}), 1.57-1.49(\mathrm{~m}, 4 \mathrm{H}), 0.86(\mathrm{~s}, 9 \mathrm{H}), 0.01(\mathrm{~s}, 6 \mathrm{H})$; ${ }^{13} \mathbf{C}$ NMR $\left(\mathrm{CDCl}_{3}, 126 \mathrm{MHz}\right): \delta=152.5(\mathrm{~d}, J=1.9 \mathrm{~Hz}), 133.0(\mathrm{~d}, J=105.0 \mathrm{~Hz}), 131.5(\mathrm{~d}$, $J=2.6 \mathrm{~Hz}), 131.2(\mathrm{~d}, J=9.8 \mathrm{~Hz}), 128.4(\mathrm{~d}, J=12.1 \mathrm{~Hz}), 122.3(\mathrm{~d}, J=103.3), 62.7(\mathrm{~s})$, $34.1(\mathrm{~d}, J=16.9 \mathrm{~Hz}), 32.28(\mathrm{~s}), 25.88$ (s), 24.26 (s), 18.25 (s), -5.37 (s); MS (EI) m/z (rel. intensity) $414\left(1,[\mathrm{M}]^{+*}\right), 399$ (3), 357 (100), 202 (10), 135 (5), 115 (3), 81 (4), 75 (8), 59 (4); HR-MS $\left(\mathrm{C}_{24} \mathrm{H}_{35} \mathrm{SiO}_{2} \mathrm{P}\right)$ : calcd. 414.2144, found 414.2162; Anal. Calcd for $\mathrm{C}_{24} \mathrm{H}_{35} \mathrm{SiO}_{2} \mathrm{P}(414.60)$ : C, 69.53; H, 8.51. Found: C, 68.99; H, 8.79.

\section{3-[(2E)-3-(diphenylphosphoryl)prop-2-enyl]-2,6-dimethyl-1H-indole (4e)}

Yellow crystalline powder; 82\% yield; m.p. 193-194 ${ }^{\circ} \mathrm{C}$; IR $\left(\mathrm{KBr}, \mathrm{cm}^{-1}\right) 3368,2961,2854,1587$, 1505, 1468, 1261, 1104, 1065, 820, 732; ${ }^{31} \mathbf{P}$ NMR $\left(\mathrm{CD}_{3} \mathrm{~S}(\mathrm{O}) \mathrm{CD}_{3}, 202 \mathrm{MHz}\right): \delta=21.4 ;{ }^{1} \mathbf{H}$ NMR $\left(\mathrm{CD}_{3} \mathrm{~S}(\mathrm{O}) \mathrm{CD}_{3}, 500 \mathrm{MHz}\right): \delta=11.70(\mathrm{~s}, 1 \mathrm{H}), 8.16(\mathrm{~d}, J=2.0 \mathrm{~Hz}, 1 \mathrm{H}), 7.80(\mathrm{dd}, J=2.1,8.8$ $\mathrm{Hz}, 1 \mathrm{H}), 7.64-7.45(\mathrm{~m}, 11 \mathrm{H}), 6.74-6.51(\mathrm{~m}, 2 \mathrm{H}), 3.77-3.70(\mathrm{~m}, 2 \mathrm{H}), 3.29(\mathrm{~s}, 3 \mathrm{H}) ;{ }^{13} \mathbf{C}$ NMR $\left(\mathrm{CD}_{3} \mathrm{~S}(\mathrm{O}) \mathrm{CD}_{3}, 126 \mathrm{MHz}\right): \delta=149.3(\mathrm{~s}), 140.9(\mathrm{~d}, J=18.3 \mathrm{~Hz}), 134.0(\mathrm{~d}, J=112.1 \mathrm{~Hz}), 133.5$ (s), 132.8 (s), 132.5 (s), 131.5 (s), 130.5 (d, $J=9.7 \mathrm{~Hz}), 128.5$ (d, $J=11.7 \mathrm{~Hz}), 122.0(\mathrm{~d}, J=$ $100.5 \mathrm{~Hz}), 117.3$ (s), 113.8 (s), 108.1 (s), 107.1 (s), 28.2 (d, $J=18 \mathrm{~Hz}), 11.6$ (s); MS (EI) $\mathrm{m} / \mathrm{z}$ (rel. intensity) $416\left(14,[\mathrm{M}]^{+\cdot}\right), 386$ (6), 215 (100), 201 (13), 198 (17), 185 (8), 168 (15), 154 (6), 127 (2), 77 (4); HR-MS $\left(\mathrm{C}_{24} \mathrm{H}_{21} \mathrm{O}_{3} \mathrm{~N}_{2} \mathrm{P}\right)$ : calcd. 416.1289, found 416.1281; Anal. Calcd for $\mathrm{C}_{24} \mathrm{H}_{21} \mathrm{O}_{3} \mathrm{~N}_{2} \mathrm{P}$ (416.42): C, 69.23; H, 5.08; N, 6.73. Found: C, 69.04; H, 5.09; N, 6.76.

\subsection{Procedure B}

To a mixture of vinylphosphine oxide $3(0.50 \mathrm{mmol})$ and $2(1.25 \mathrm{mmol})$ in $\mathrm{CH}_{2} \mathrm{Cl}_{2}(4 \mathrm{~mL})$ was added a solution of catalyst $1(0.025 \mathrm{mmol}, 5 \mathrm{~mol} \%)$ in $\mathrm{CH}_{2} \mathrm{Cl}_{2}(1 \mathrm{~mL})$. The resulting mixture was stirred at $45{ }^{\circ} \mathrm{C}$ for $16 \mathrm{~h}$. The solvent was removed under reduced pressure. The crude product 4 was purified by flash chromatography using the eluent system as above.

\section{(2E)-3-(diphenylphosphoryl)prop-2-enyl acetate (4b)}

Pale brown oil; 52\% (NMR). IR (film, $\mathrm{cm}^{-1}$ ) 3054, 3023, 2997, 2955, 2934, 1741, 1639, 1437 , 1386, 1232, 1180, 1120, 1105, 1072, 1029, 753, 734, 723, 695, 611, 530; ${ }^{31} \mathbf{P}$ NMR $\left(\mathrm{CDCl}_{3}, 202\right.$ MHz): $\delta=24.9 ;{ }^{1} \mathbf{H} \mathbf{~ N M R}\left(\mathrm{CDCl}_{3}, 500 \mathrm{MHz}\right): \delta=7.73-7.64(\mathrm{~m}, 4 \mathrm{H}), 7.49-7.44(\mathrm{~m}, 6 \mathrm{H})$, 6.74 (ddt, $J=19.5,17.1,4.2 \mathrm{~Hz}, 1 \mathrm{H}$ ), 6.49 (ddt, $J=22.7,17.1,1.9 \mathrm{~Hz}, 1 \mathrm{H}$ ), $4.80-4.77$ (m, $2 \mathrm{H}), 2.11(\mathrm{~s}, 3 \mathrm{H}) ;{ }^{13} \mathbf{C ~ N M R}\left(\mathrm{CDCl}_{3}, 126 \mathrm{MHz}\right): \delta=170.0(\mathrm{~s}), 144.4(\mathrm{~d}, J=3.4 \mathrm{~Hz}), 132.2$ $(\mathrm{d}, J=106.0 \mathrm{~Hz}), 131.7(\mathrm{~d}, J=2.8 \mathrm{~Hz}), 131.0(\mathrm{~d}, J=10.1 \mathrm{~Hz}), 128.6(\mathrm{~d} . J=12.2 \mathrm{~Hz}), 123.0$ $(\mathrm{d}, J=101.4 \mathrm{~Hz}), 63.6(\mathrm{~d}, J=17.8 \mathrm{~Hz}), 20.7(\mathrm{~s})$; HPLC-MS $\left(\mathrm{H}_{2} \mathrm{O} 50 \%, \mathrm{CH}_{3} \mathrm{OH} 35 \%, \mathrm{CH}_{3} \mathrm{CN}\right.$ $15 \%, 0.2 \mathrm{ml} / \mathrm{min}$ ): retention time $12.5 \mathrm{~min}$; MS (ESI) $\mathrm{m} / z$ (rel. intensity) $301(30)[\mathrm{M}+\mathrm{H}]^{+}, 323$ (100) $[\mathrm{M}+\mathrm{Na}]^{+}, 339(25)[\mathrm{M}+\mathrm{K}]^{+}$; HR-MS $\left(\mathrm{C}_{17} \mathrm{H}_{17} \mathrm{O}_{3} \mathrm{PNa}\right)$ : calcd. 323.0808, found 323.0817.

\section{[(1E)-3-chloroprop-1-enyl](diphenyl)phosphine oxide (4c):}

White crystalline powder; 65\% yield; m.p. 233-234 ${ }^{\circ} \mathrm{C}$; IR $\left(\mathrm{KBr}, \mathrm{cm}^{-1}\right) 3055,3013,2933$, 1626, 1483, 1440, 1416, 1291, 1180, 1167, 1121, 1105, 1073, 995, 834, 754, 697, 570, 525; ${ }^{31} \mathbf{P}$ NMR $\left(\mathrm{CDCl}_{3}, 202 \mathrm{MHz}\right): \delta=24.1 ;{ }^{1} \mathbf{H ~ N M R}\left(\mathrm{CDCl}_{3}, 500 \mathrm{MHz}\right): \delta=7.65-7.61(\mathrm{~m}, 4 \mathrm{H})$, $7.42-7.40(\mathrm{~m}, 6 \mathrm{H}), 6.77$ (ddt, $J=18.4,16.6,5.1 \mathrm{~Hz}, 1 \mathrm{H}), 6.57$ (ddt, $J=22.5,16.6,1.6 \mathrm{~Hz}, 1$ 
$\mathrm{H}), 4.18(\mathrm{dt}, J=5.0,2.0 \mathrm{~Hz}, 2 \mathrm{H}) ;{ }^{13} \mathbf{C ~ N M R}\left(\mathrm{CDCl}_{3}, 126 \mathrm{MHz}\right): \delta=144.9(\mathrm{~d}, J=4.2 \mathrm{~Hz})$, $132.5(\mathrm{~d}, J=106.3 \mathrm{~Hz}), 132.0(\mathrm{~d}, J=2.6 \mathrm{~Hz}), 131.3(\mathrm{~d}, J=9.9 \mathrm{~Hz}), 128.7(\mathrm{~d}, J=12.2 \mathrm{~Hz})$, $125.4(\mathrm{~d}, J=100.1 \mathrm{~Hz}$ ), 44.2 (d, $J=19.6 \mathrm{~Hz}$ ); MS (ESI) $\mathrm{m} / z$ (rel. intensity) 277 (25) $[\mathrm{M}+$ $\mathrm{H}]^{+}, 299(100)[\mathrm{M}+\mathrm{Na}]^{+}$; HR-MS $\left(\mathrm{C}_{15} \mathrm{H}_{14} \mathrm{ClOPNa}\right)$ : calcd. 299.0363, found 299.0376.

\section{[(1E)-6-bromohex-1-enyl](diphenyl)phosphine oxide (4d):}

Pale gray crystalline powder; 99\% yield; m.p. 77-79 ${ }^{\circ} \mathrm{C}$; IR $\left(\mathrm{KBr}, \mathrm{cm}^{-1}\right)$ 3053, 2991, 2939, 1627, 1591, 1485, 1437, 1247, 1180, 1120, 997, 804, 719, 696, 548, 536; ${ }^{31} \mathbf{P} \mathbf{N M R}\left(\mathrm{CDCl}_{3}, 202\right.$ MHz): $\delta=24.3 ;{ }^{1} \mathbf{H}$ NMR $\left(\mathrm{CDCl}_{3}, 500 \mathrm{MHz}\right): \delta=7.78-7.67(\mathrm{~m}, 4 \mathrm{H}), 7.54-7.44(\mathrm{~m}, 6 \mathrm{H}), 6.73$ (ddt, $J=19.4,17.0,6.5 \mathrm{~Hz}, 1 \mathrm{H}$ ), 6.27 (ddt, $J=24.2,17.0,1.5 \mathrm{~Hz}, 1 \mathrm{H}$ ), 3.40 (t, $J=6.6 \mathrm{~Hz}, 2$ $\mathrm{H}), 2.34(\mathrm{dd}, J=14.4,7.2 \mathrm{~Hz}, 2 \mathrm{H}), 1.92-1.86(\mathrm{~m}, 2 \mathrm{H}), 1.69-1.63(\mathrm{~m}, 2 \mathrm{H}) ;{ }^{13} \mathbf{C} \mathbf{N M R}\left(\mathrm{CDCl}_{3}\right.$, $126 \mathrm{MHz}): \delta=151.5(\mathrm{~d}, J=1.9 \mathrm{~Hz}), 133.0(\mathrm{~d}, J=104.4 \mathrm{~Hz}), 131.7(\mathrm{~d}, J=2.6 \mathrm{~Hz}), 131.3(\mathrm{~d}$, $J=9.9 \mathrm{~Hz}), 128.5(\mathrm{~d}, J=12.1 \mathrm{~Hz}), 122.5(\mathrm{~d}, J=102.6), 33.5(\mathrm{~d}, J=16.9 \mathrm{~Hz}), 33.16$ (s), 32.07 (s), 26.41 (s); MS (ESI) $m / z$ (rel. intensity) $363(30)[\mathrm{M}+\mathrm{H}]^{+}, 385(100)[\mathrm{M}+\mathrm{Na}]^{+}$; HR-MS $\left(\mathrm{C}_{18} \mathrm{H}_{20} \mathrm{BrOPNa}\right)$ : calcd. 385.0327, found 385.0346.

\section{Diphenyl[(1E)-prop-1-enyl]phosphine oxide ${ }^{7}(4 \mathbf{f})$ :}

To a mixed suspension of $\mathbf{3 a}(0.5 \mathrm{mmol})$ in 2-methyl-2-butene $(5 \mathrm{~mL})$ was added solution $\mathbf{1 c}$ ( $5 \mathrm{~mol} \%$; $0.025 \mathrm{mmol}$ ) in $\mathrm{CH}_{2} \mathrm{Cl}_{2}(0.2 \mathrm{~mL})$. The resulting mixture was refluxed for $16 \mathrm{~h}$. After evaporation of solvent, the residue was purified by flash chromatography (hexane-acetone $4: 1$, then hexane-ethyl acetate-methanol 5:2:1) affording the title compound as a pale gray crystalline powder, 98\% yield. M.p. $128-129{ }^{\circ} \mathrm{C}$; IR $\left(\mathrm{KBr}, \mathrm{cm}^{-1}\right)$ 3053, 2995, 2940, 1632, 1590, 1485, 1439, 1189, $1120,997,795,743,718,693,549,528 ;{ }^{31} \mathbf{P}$ NMR $\left(\mathrm{CDCl}_{3}, 202 \mathrm{MHz}\right): \delta=24.7 ;{ }^{1} \mathbf{H} \mathbf{~ N M R}\left(\mathrm{CDCl}_{3}\right.$, $500 \mathrm{MHz}): \delta=7.73-7.66(\mathrm{~m}, 4 \mathrm{H}), 7.54-7.42(\mathrm{~m}, 6 \mathrm{H}), 6.71(\mathrm{ddq}, J=19.6,16.9,6.6 \mathrm{~Hz}, 1 \mathrm{H}), 6.27$ $(\mathrm{ddq}, J=24.2,16.9,1.6 \mathrm{~Hz}, 1 \mathrm{H}), 1.99(\mathrm{dt}, J=6.6,1.9 \mathrm{~Hz}) ;{ }^{13} \mathbf{C} \mathbf{N M R}\left(\mathrm{CDCl}_{3}, 126 \mathrm{MHz}\right): \delta=$ $147.8(\mathrm{~d}, J=2.5 \mathrm{~Hz}), 133.0(\mathrm{~d}, J=104.9 \mathrm{~Hz}), 131.6(\mathrm{~d}, J=2.6 \mathrm{~Hz}), 131.2(\mathrm{~d}, J=9.8 \mathrm{~Hz}), 128.4$ $(\mathrm{d}, J=12.1 \mathrm{~Hz}), 123.5(\mathrm{~d}, J=103.6 \mathrm{~Hz}), 20.3(\mathrm{~d}, J=18.5 \mathrm{~Hz}) ; \mathbf{M S}(\mathbf{E S I}) \mathrm{m} / z$ (rel. intensity) $243(70)[\mathrm{M}+\mathrm{H}]^{+}, 265(100)[\mathrm{M}+\mathrm{H}]^{+}$; HR-MS $\left(\mathrm{C}_{15} \mathrm{H}_{15} \mathrm{OPNa}\right)$ : calcd. 265.0753, found 265.0771.

\section{(2-methylprop-1-enyl)(diphenyl)phosphine oxide ${ }^{8}(4 \mathrm{~g})$ :}

Light grey solid (in a mixture with 4f; 30\% (NMR). IR (film, $\mathrm{cm}^{-1}$ ) 3053, 3022, 2993, 2964, 2910, 2849, 1631, 1589, 1485, 1439, 1181, 1120, 1104, 1071, 996, 971, 794, 754, 743, 720, 694, 610, 563, 550, 527, 501; ${ }^{31} \mathbf{P}$ NMR $\left(\mathrm{CDCl}_{3}, 202 \mathrm{MHz}\right): \delta=25.3 ;{ }^{1} \mathbf{H}$ NMR $\left(\mathrm{CDCl}_{3}, 500 \mathrm{MHz}\right)$ : $\delta=7.73-7.66(\mathrm{~m}, 4 \mathrm{H}), 7.54-7.42(\mathrm{~m}, 6 \mathrm{H}), 5.89\left(\mathrm{dm}, J_{P-H}=25.4 \mathrm{~Hz}(H-H\right.$ fine splitting, line separation $0.3 \mathrm{~Hz}), 1 \mathrm{H}) 2.09\left(\mathrm{dd}, J_{H-H}=1 \mathrm{~Hz}, J_{H-P}=2 \mathrm{~Hz}, 3 \mathrm{H}\right), 2.07(\mathrm{br} \mathrm{s}, 3 \mathrm{H}) ; \mathbf{M S}$ (ESI) $\mathrm{m} / z$ (rel. intensity) $243(100) 257(10)[\mathrm{Na}+\mathrm{H}]^{+}, 279(5)[\mathrm{M}+\mathrm{Na}]^{+}$; HPLC-MS $\left(\mathrm{H}_{2} \mathrm{O}\right.$ $\left.57 \%, \mathrm{CH}_{3} \mathrm{OH} 43 \%, 0.2 \mathrm{ml} / \mathrm{min}\right)$ : retention time = $83.5 \mathrm{~min}$; HR-MS $\left(\mathrm{C}_{16} \mathrm{H}_{18} \mathrm{OP}\right):$ calcd. 257.1090, found 257.1102.

$\left(S_{\mathrm{P}}\right)$-(-)-[(1E)-6-bromohex-1-enyl](methyl)phenylphosphine oxide (4h):

Pale brown oil; $86 \%$ yield; $[\alpha]_{\mathrm{D}}^{20}-28.9 \mathrm{deg}\left(c \mathrm{1.32}, \mathrm{CH}_{2} \mathrm{Cl}_{2}\right)$; IR (film, $\left.\mathrm{cm}^{-1}\right)$ 2936, 1629, 1437, 1294, 1181, 1115, 980, 896, 742, 696, 502; ${ }^{31} \mathbf{P} \mathbf{~ N M R}\left(\mathrm{CDCl}_{3}, 202 \mathrm{MHz}\right): \delta=27.4 ;{ }^{1} \mathbf{H}$ NMR $\left(\mathrm{CDCl}_{3}, 500 \mathrm{MHz}\right): \delta=7.81-7.69(\mathrm{~m}, 2 \mathrm{H}), 7.54-7.44(\mathrm{~m}, 3 \mathrm{H}), 6.66(\mathrm{ddt}, J=19.4,17.0$,

7 M.-P. Teulade, P. Savignac, J. Organomet. Chem., 1986, 304, 283.

8 (a) P.F. Cann, D. Howells, S. Warren, J. Chem. Soc. Perkin Trans. 2; 1972; 304; (b) A. E. Antoshin, V. I. Evreinov, A. V. Kharitonov, A. N. Pushin, A. N. Yarkevich, Bull. Acad. Sci. USSR Div. Chem. Sci. 1991, 40, 1647; (Izv. Akad. Nauk SSSR Ser. Khim., 1991, 8, 1860). 
$6.5 \mathrm{~Hz}, 1 \mathrm{H}), 6.02(\mathrm{ddt}, J=24.9,17.0,1.6 \mathrm{~Hz}, 1 \mathrm{H}), 3.39(\mathrm{t}, J=6.7,2 \mathrm{H}), 2.31-2.24(\mathrm{~m}, 2$ $\mathrm{H}), 1.92-1.83(\mathrm{~m}, 2 \mathrm{H}), 1.75(\mathrm{~d}, J=13.2 \mathrm{~Hz}, 3 \mathrm{H}), 1.65-1.58(\mathrm{~m}, 2 \mathrm{H}) ;{ }^{13} \mathbf{C} \mathbf{N M R}\left(\mathrm{CDCl}_{3}, 126\right.$ MHz): $\delta=149.8(\mathrm{~d}, J=1.7 \mathrm{~Hz}), 134(\mathrm{~d}, J=90.6 \mathrm{~Hz}), 131.6(\mathrm{~d}, J=2.7 \mathrm{~Hz}), 130.0(\mathrm{~d}, 9.7 \mathrm{~Hz})$, 128.6 (d, $J=11.8 \mathrm{~Hz}), 124.0(\mathrm{~d}, J=99.6 \mathrm{~Hz}), 33.3$ (d, $J=16.8 \mathrm{~Hz}), 33.2$ (s), 32.0 (s), 26.4 (d, $J=1.1 \mathrm{~Hz}$ ), 17.0 (d, $J=74.5 \mathrm{~Hz}$ ); MS (ESI) $m / z$ (rel. intensity) 301 (12) $[\mathrm{M}+\mathrm{H}]^{+} ; 323$ (100) $[\mathrm{M}+\mathrm{Na}]^{+}$; HR-MS $\left(\mathrm{C}_{13} \mathrm{H}_{18} \mathrm{BrOPNa}\right)$ : calcd. 323.0171, found 323.0168.

$\left(S_{\mathrm{P}}\right)$-(-)-(5E)-6-[methyl(phenyl)phosphoryl]hex-5-en-2-one (4i):

Pale brown oil; $85 \%$ yield; $[\alpha]_{\mathrm{D}}^{20}-44.7 \mathrm{deg}\left(c 1.35, \mathrm{CH}_{2} \mathrm{Cl}_{2}\right)$; IR (film, $\left.\mathrm{cm}^{-1}\right)$ 3055, 2993, 2914, 1714, 1629, 1591, 1484, 1437, 1366, 1296, 1179, 1115, 985, 894, 744, 698, 512; ${ }^{31} \mathbf{P}$ NMR $\left(\mathrm{CDCl}_{3}, 202 \mathrm{MHz}\right): \delta=27.3 ;{ }^{1} \mathbf{H} \mathbf{N M R}\left(\mathrm{CDCl}_{3}, 500 \mathrm{MHz}\right): \delta=7.72-7.66(\mathrm{~m}, 2 \mathrm{H}), 7.54-7.45(\mathrm{~m}$, $3 \mathrm{H}), 6.64$ (ddt, $J=19.2,17.0,6.5 \mathrm{~Hz}, 1 \mathrm{H}$ ), 6.03 (ddt, $J=24.4,17.0,1.3 \mathrm{~Hz}, 1 \mathrm{H}$ ), 2.62-2.46 $(\mathrm{m}, 4 \mathrm{H}), 2.14(\mathrm{~s}, 3 \mathrm{H}), 1.74(\mathrm{~d}, J=13.2,3 \mathrm{H}) ;{ }^{13} \mathbf{C ~ N M R}\left(\mathrm{CDCl}_{3}, 126 \mathrm{MHz}\right): \delta=206.7$ (s), $148.6(\mathrm{~d}, J=1.9 \mathrm{~Hz}), 133.9(\mathrm{~d}, J=102.2 \mathrm{~Hz}), 131.6(\mathrm{~d}, J=2.6 \mathrm{~Hz}), 130.0(\mathrm{~d}, J=9.7 \mathrm{~Hz})$, $128.6(\mathrm{~d}, J=11.8 \mathrm{~Hz}), 124.4(\mathrm{~d}, J=99.2 \mathrm{~Hz}), 41.4(\mathrm{~d}, J=1.0 \mathrm{~Hz}), 29.9(\mathrm{~s}), 28.0(\mathrm{~d}, J=17.2$ $\mathrm{Hz}), 16.9$ (d, $J=74.7 \mathrm{~Hz}$ ); MS (ESI) $m / z$ (rel. intensity) $237(30)[\mathrm{M}+\mathrm{H}]^{+}, 259(100)[\mathrm{M}+$ $\mathrm{Na}]^{+}$; HR-MS $\left(\mathrm{C}_{13} \mathrm{H}_{17} \mathrm{O}_{2} \mathrm{PNa}\right)$ : calcd. 259.0858, found 259.0848.

\section{Methyl $\left(S_{\mathrm{P}}\right)$-(-)-(10E)-11-(methyl(phenyl)phosphoryl)undec-10-enoate (4k):}

Pale brown oil; 74\% yield; $[\alpha]_{\mathrm{D}}^{20}-26.2 \mathrm{deg}\left(c 1.5, \mathrm{CH}_{2} \mathrm{Cl}_{2}\right)$; IR (film, $\left.\mathrm{cm}^{-1}\right)$ 2929, 2855, 1736, 1629, 1437, 1294, 1179, 1115, 984, 895, 743, 697, 506; ${ }^{31} \mathbf{P}$ NMR $\left(\mathrm{CDCl}_{3}, 202 \mathrm{MHz}\right): \delta=27.5$; ${ }^{1} \mathbf{H}$ NMR $\left(\mathrm{CDCl}_{3}, 500 \mathrm{MHz}\right): \delta=7.72-7.68(\mathrm{~m}, 2 \mathrm{H}, P h), 7.49-7.46(\mathrm{~m}, 3 \mathrm{H}, P h), 6.67$ (ddt, $J=$ 19.5, 17.0, $6.6 \mathrm{~Hz}, 1 \mathrm{H}), 5.98(\mathrm{ddt}, J=25.1,17.0,1.4 \mathrm{~Hz}, 1 \mathrm{H}), 3.65(\mathrm{~s}, 3 \mathrm{H}), 2.28(\mathrm{t}, J=7.5$ $\mathrm{Hz}, 2 \mathrm{H}), 2.25-2.19(\mathrm{~m}, 2 \mathrm{H}), 1.74(\mathrm{~d}, J=13.2 \mathrm{~Hz}, 3 \mathrm{H}), 1.63-1.55(\mathrm{~m}, 2 \mathrm{H}), 1.47-1.39$ (m, 2 $\mathrm{H}), 1.33-1.23(\mathrm{~m}, 8 \mathrm{H}) ;{ }^{13} \mathbf{C} \mathbf{N M R}\left(\mathrm{CDCl}_{3}, 126 \mathrm{MHz}\right): \delta=174.2(\mathrm{~s}), 150.8(\mathrm{~d}, J=1.4 \mathrm{~Hz}), 134.3$ $(\mathrm{d}, J=101.5 \mathrm{~Hz}), 131.5(\mathrm{~d}, J=2.6 \mathrm{~Hz}), 130.0(\mathrm{~d}, J=9.7 \mathrm{~Hz}), 128.6(\mathrm{~d}, J=11.8 \mathrm{~Hz}), 123.1$ (d, $J=100.2 \mathrm{~Hz}), 51.4(\mathrm{~s}), 34.3(\mathrm{~d}, J=16.7 \mathrm{~Hz}), 34.0(\mathrm{~s}), 29.1(\mathrm{~s}), 29.0(\mathrm{~s}), 28.9$ (s), 28.8 (s), 27.8 (s), 24.9 (s), 16.9 (d, $J=74.4 \mathrm{~Hz}$ ); MS (ESI) $m / z$ (rel. intensity) 337 (90) $[\mathrm{M}+\mathrm{H}]^{+}, 359$ (100) $[\mathrm{M}+\mathrm{Na}]^{+}$; HR-MS $\left(\mathrm{C}_{19} \mathrm{H}_{29} \mathrm{O}_{3} \mathrm{PNa}\right)$ : calcd. 359.1747, found 359.1765.

\section{$\left(R_{\mathrm{P}}\right)$-((-)-tert-Butyl(phenyl)[(1E)-3-phenylprop-1-enyl]phosphine oxide (4I):}

Pale gray crystals; $72 \%$; $[\alpha]_{\mathrm{D}}^{20}-27.6 \mathrm{deg}\left(c\right.$ 1, $\left.\mathrm{CH}_{2} \mathrm{Cl}_{2}\right) ;$ m.p. $103-104{ }^{\circ} \mathrm{C} ; \mathbf{I R}\left(\mathrm{KBr}, \mathrm{cm}^{-1}\right)$ 2961, 1943, 1732, 1668, 1628, 1603, 1495, 1476, 1436, 1364, 1268, 1213, 1213, 1171, 1110, 997, 816, 776, 748, 699; ${ }^{31} \mathbf{P}$ NMR $\left(\mathrm{CDCl}_{3}, 202 \mathrm{MHz}\right): \delta=38.8 ;{ }^{1} \mathbf{H}$ NMR $\left(\mathrm{CDCl}_{3}, 500 \mathrm{MHz}\right): \delta=$ 7.72-7.16 (m, $10 \mathrm{H}, P h), 7.07$ (tt, $J=16.9,6.3 \mathrm{~Hz}, 1 \mathrm{H}, \mathrm{CH}_{2}-\mathrm{CH}$ ), 6.23 (ddt, $J=26.9,16.9$, $1.7 \mathrm{~Hz}, 1 \mathrm{H}, \mathrm{P}(\mathrm{O})-\mathrm{CH}$ ), $3.63\left(\mathrm{dt}, J=6.3,1.9 \mathrm{~Hz}, 2 \mathrm{H}, \mathrm{Ph}-\mathrm{CH}_{2}\right), 1.09(\mathrm{~d}, J=14.9 \mathrm{~Hz}, 9 \mathrm{H}$, $\left.\mathrm{C}-\left(\mathrm{CH}_{3}\right)_{3}\right) ;{ }^{13} \mathbf{C} \mathbf{N M R}\left(\mathrm{CDCl}_{3}, 126 \mathrm{MHz}\right): \delta=151.7\left(\mathrm{~s}, \mathrm{CH}_{2}-\mathrm{CH}\right), 137.8\left(\mathrm{~s}, \mathrm{CH}_{2}-C\right), 131.8(\mathrm{~d}$, $J=8 \mathrm{~Hz}, o-C$ in $\mathrm{Ph}-\mathrm{P}(\mathrm{O})), 131.3(\mathrm{~d}, J=2.6 \mathrm{~Hz}, p-C$ in $\mathrm{Ph}-\mathrm{P}(\mathrm{O})), 130.8(\mathrm{~d}, J=92.8 \mathrm{~Hz}, \mathrm{P}(\mathrm{O})-C)$, $128.9\left(\mathrm{~s}, o-C\right.$ in $\left.\mathrm{Ph}-\mathrm{CH}_{2}\right), 128.6\left(\mathrm{~s}, m-C\right.$ in $\left.\mathrm{Ph}-\mathrm{CH}_{2}\right), 128.1(\mathrm{~d}, J=10.9 \mathrm{~Hz}, m-C$ in $\mathrm{Ph}-\mathrm{P}(\mathrm{O})$ ), $126.6\left(\mathrm{~s}, p-C\right.$ in $\left.\mathrm{Ph}-\mathrm{CH}_{2}\right), 119.1(\mathrm{~d}, J=91.5 \mathrm{~Hz}, C \mathrm{H}-\mathrm{P}(\mathrm{O})), 40.8\left(\mathrm{~d}, J=15.5 \mathrm{~Hz}, C \mathrm{H}_{2}\right), 32.6$ $\left(\mathrm{d}, J=73.3 \mathrm{~Hz}, C\left(\mathrm{CH}_{3}\right)_{3}\right), 24.2\left(\mathrm{~s}, \mathrm{C}\left(\mathrm{CH}_{3}\right)_{3}\right)$; MS (ESI) $\mathrm{m} / z$ (rel. intensity) $299(70)[\mathrm{M}+\mathrm{H}]^{+}$, 321 (100) $[\mathrm{M}+\mathrm{Na}]^{+}$; HR-MS $\left(\mathrm{C}_{19} \mathrm{H}_{23} \mathrm{OPNa}\right)$ : calcd. 321.1379, found 321.1393.

$\left(S_{\mathrm{P}}, S_{\mathrm{P}}\right)$-(-)-(E)-ethene-1,2-diylbis[methyl(phenyl)phosphine] $\operatorname{dioxide}^{9}(5)$ :

To a mixed solution of $\mathbf{3 b}(0.5 \mathrm{mmol})$ in $\mathrm{CH}_{2} \mathrm{Cl}_{2}(4 \mathrm{~mL})$ was added solution $1 \mathbf{c}(0.025 \mathrm{mmol})$

\footnotetext{
9 K. M. Pietrusiewicz, W. Wiśniewski, M. Zabłocka, Tetrahedron 1989, 45337.
} 
in $\mathrm{CH}_{2} \mathrm{Cl}_{2}(1 \mathrm{~mL})$. The resulting solution was refluxed for $16 \mathrm{~h}$. Solvent was evaporated and the crude residue was purified using flash chromatography (hexane-ethyl acetate-methanol 5:2:0.5 then $\mathrm{CH}_{2} \mathrm{Cl}_{2}$-methanol 10:1) to afford the title compound as a white crystalline powder, $90 \%$ yield. $[\alpha]_{\mathrm{D}}^{20}$ -255.0 deg ( $\left.c 1, \mathrm{CH}_{2} \mathrm{Cl}_{2}\right)$; m.p. $238-239{ }^{\circ} \mathrm{C}$; $\mathbf{I R}\left(\mathrm{KBr}, \mathrm{cm}^{-1}\right)$ 3054, 2989, 2904, 1838, 1590, 1482, 1437, 1301, 1177, 1113, 1025, 894, 882, 754, 740, 692, 484; ${ }^{31} \mathbf{P}$ NMR $\left(\mathrm{CDCl}_{3}, 202 \mathrm{MHz}\right): \delta$ $=26.4 ;{ }^{1} \mathbf{H} \mathbf{N M R}\left(\mathrm{CDCl}_{3}, 500 \mathrm{MHz}\right)$, (second order spectrum $): \delta=7.68-7.62(\mathrm{~m}, 4 \mathrm{H}), 7.53-742$ $(\mathrm{m}, 6 \mathrm{H}), 7.33\left(\mathrm{t}, J_{A X}+J_{B X}=50 \mathrm{~Hz}, 2 \mathrm{H}\right), 1.83$ (filled-in doublet, line separation $=13.1 \mathrm{~Hz}, 6$ $\mathrm{H}) ;{ }^{13} \mathbf{C ~ N M R}\left(\mathrm{CDCl}_{3}, 126 \mathrm{MHz}\right)$, (second order spectrum, only the central of multiplet signals are listed): $\delta=141.8$ (6 lines), 132.2 (s), 132 (6 lines), 130.0 (3 lines), 128.9 (3 lines), 16.7 (6 lines); MS (ESI) $m / z$ (rel. intensity) $305(25)[\mathrm{M}+\mathrm{H}]^{+}, 327(100)[\mathrm{M}+\mathrm{Na}]^{+}$; HR-MS $\left(\mathrm{C}_{16} \mathrm{H}_{18} \mathrm{O}_{2} \mathrm{P}_{2} \mathrm{Na}\right)$ : calcd. 327.0674, found 327.0690.

$\left(S_{\mathrm{P}}\right)$-(-)-[(E)-2-(2-isopropoxy-5-nitrophenyl)vinyl](methyl)phenylphosphine oxide (6):

Brown low-melting solid, 5\% yield; $[\alpha]_{\mathrm{D}}^{20}-29.2 \mathrm{deg}\left(c 1, \mathrm{CH}_{2} \mathrm{Cl}_{2}\right)$; m.p. 103-104 ${ }^{\circ} \mathrm{C}$; IR (film from $\mathrm{CH}_{2} \mathrm{Cl}_{2}, \mathrm{~cm}^{-1}$ ) 3057, 2981, 2927, 2854, 1916, 1731, 1608, 1582, 1514, 1482, 1342, 1274, 1181, 1105, 952, 897, 744, 696; ${ }^{31} \mathbf{P}$ NMR $\left(\mathrm{CDCl}_{3}, 202 \mathrm{MHz}\right): \delta=28.1 ;{ }^{1} \mathbf{H} \mathbf{~ N M R}\left(\mathrm{CDCl}_{3}, 500\right.$ $\mathrm{MHz}): \delta=8.39(\mathrm{~d}, J=2.8 \mathrm{~Hz}, 1 \mathrm{H}), 8.20(\mathrm{dd}, J=9.2,2.8 \mathrm{~Hz}, 1 \mathrm{H}), 7.82-7.76(\mathrm{~m}, 2 \mathrm{H}), 7.74$ $(\mathrm{dd}, J=20.0,17.6 \mathrm{~Hz}, 1), 7.58-7.48(\mathrm{~m}, 3 \mathrm{H}), 6.95(\mathrm{~d}, J=9.2 \mathrm{~Hz}, 1 \mathrm{H}), 6.78(\mathrm{dd}, J=23.5$, $17.5 \mathrm{~Hz}, 1 \mathrm{H}), 4.73$ (spt, $J=6.0 \mathrm{~Hz}, 1 \mathrm{H}), 1.89(\mathrm{~d}, J=13.2 \mathrm{~Hz}, 3 \mathrm{H}), 1.41$ (dd, $J=6.0,2.3$ $\mathrm{Hz}, 6 \mathrm{H}) ;{ }^{13} \mathbf{C ~ N M R}\left(\mathrm{CDCl}_{3}, 126 \mathrm{MHz}\right): \delta=160.8$ (s), 140.9 (s), 139.0 (d, $\left.J=4.5 \mathrm{~Hz}\right), 133.5$ (d, $J=102.9 \mathrm{~Hz}), 130.1(\mathrm{~d}, J=9.7 \mathrm{~Hz}), 128.8(\mathrm{~d}, J=11.8 \mathrm{~Hz}), 126.4(\mathrm{~s}), 125.4(\mathrm{~d}, J=18.0 \mathrm{~Hz})$, $123.9(\mathrm{~d}, J=99.0 \mathrm{~Hz}), 123.6(\mathrm{~s}), 112.6(\mathrm{~s}), 72.1(\mathrm{~s}), 21.8(\mathrm{~s}), 16.9$ (d, $J=75.2 \mathrm{~Hz})$; MS (ESI) $m / z$ (rel. intensity) $346(100)[\mathrm{M}+\mathrm{H}]^{+}, 368(70)[\mathrm{M}+\mathrm{Na}]^{+}$; HR-MS $\left(\mathrm{C}_{18} \mathrm{H}_{20} \mathrm{O}_{4} \mathrm{NPNa}\right)$ : calcd. 368.1022 , found 368.1039 .

\section{Determination of the optical purity of $3 c$ and 41}

The optical purity of $\mathbf{3 c}$ and $\mathbf{4 l}$ were calculated from the corresponding ${ }^{31} \mathrm{P},{ }^{1} \mathrm{H}$ NMR spectra registered in the presence of 2 eq. of (S)- $N$-[1-(1-naphthyl)ethyl]-3,5-dinitrobenzamide.

3c: $[\alpha]_{\mathrm{D}}^{20}-57.2 \operatorname{deg}\left(c 1, \mathrm{CH}_{2} \mathrm{Cl}_{2}\right) ;{ }^{31} \mathbf{P} \mathbf{N M R}\left(\mathrm{CDCl}_{3}, 202 \mathrm{MHz}\right): \delta$ (rel. weight) $=39.3(1.00)$, 39.26 (0.16); significant fragments of ${ }^{1} \mathbf{H}$ NMR $\left(\mathrm{CDCl}_{3}, 500 \mathrm{MHz}\right): \delta$ (rel. weight) $=6.46(0.13)$, 6.45 (1.00); 1.15 (0.15), 1.13 (1.00); 1.11 (0.15), 1.09 (1.00). Calculated optical purity: $73 \pm 3 \%$ ee.

4I: $[\alpha]_{\mathrm{D}}^{20}-27.6 \mathrm{deg}\left(c 1, \mathrm{CH}_{2} \mathrm{Cl}_{2}\right) ;{ }^{31} \mathbf{P} \mathbf{N M R}\left(\mathrm{CDCl}_{3}, 202 \mathrm{MHz}\right): \delta$ (rel. weight $)=39.30(1.00)$, 39.25 (0.15); significant fragments of ${ }^{1} \mathbf{H}$ NMR $\left(\mathrm{CDCl}_{3}, 500 \mathrm{MHz}\right): \delta$ (rel. weight) $=6.29(0.15)$, 6.27 (1.00); 6.26 (0.16), 6.24 (1.00); 6.20 (0.17), 6.19 (1.00); 1.10 (0.14), 1.08 (1.00); 1.07 (0.16), 1.05 (1.00). Calculated optical purity: $72 \pm 4 \%$ ee. 


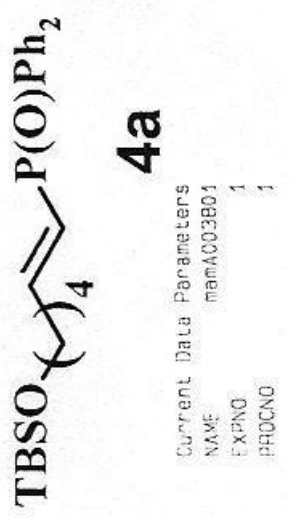

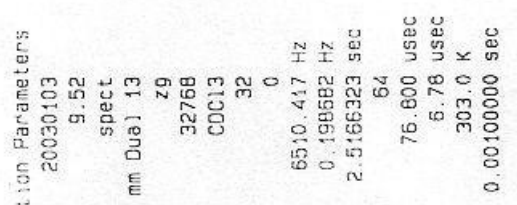

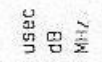

$\frac{5}{\frac{1}{2}} \pm$

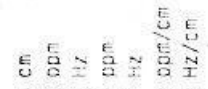

요의

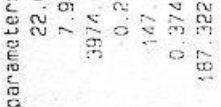

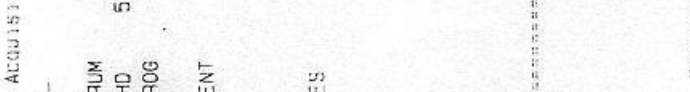

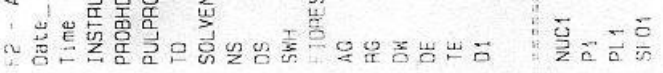

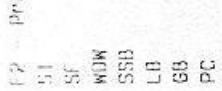
$\frac{\vec{s}}{a}$

等

S10.0-

$\nabla 905^{\circ} \mathrm{T}$

$890^{\circ} 0$

टZEट 0

$898^{\circ} 0$

$\checkmark 98^{\circ}$

OจマE.

$O \angle E O$

$125^{\circ} 1$

B25

$\forall E G$.

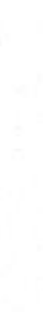

O6S' $E$
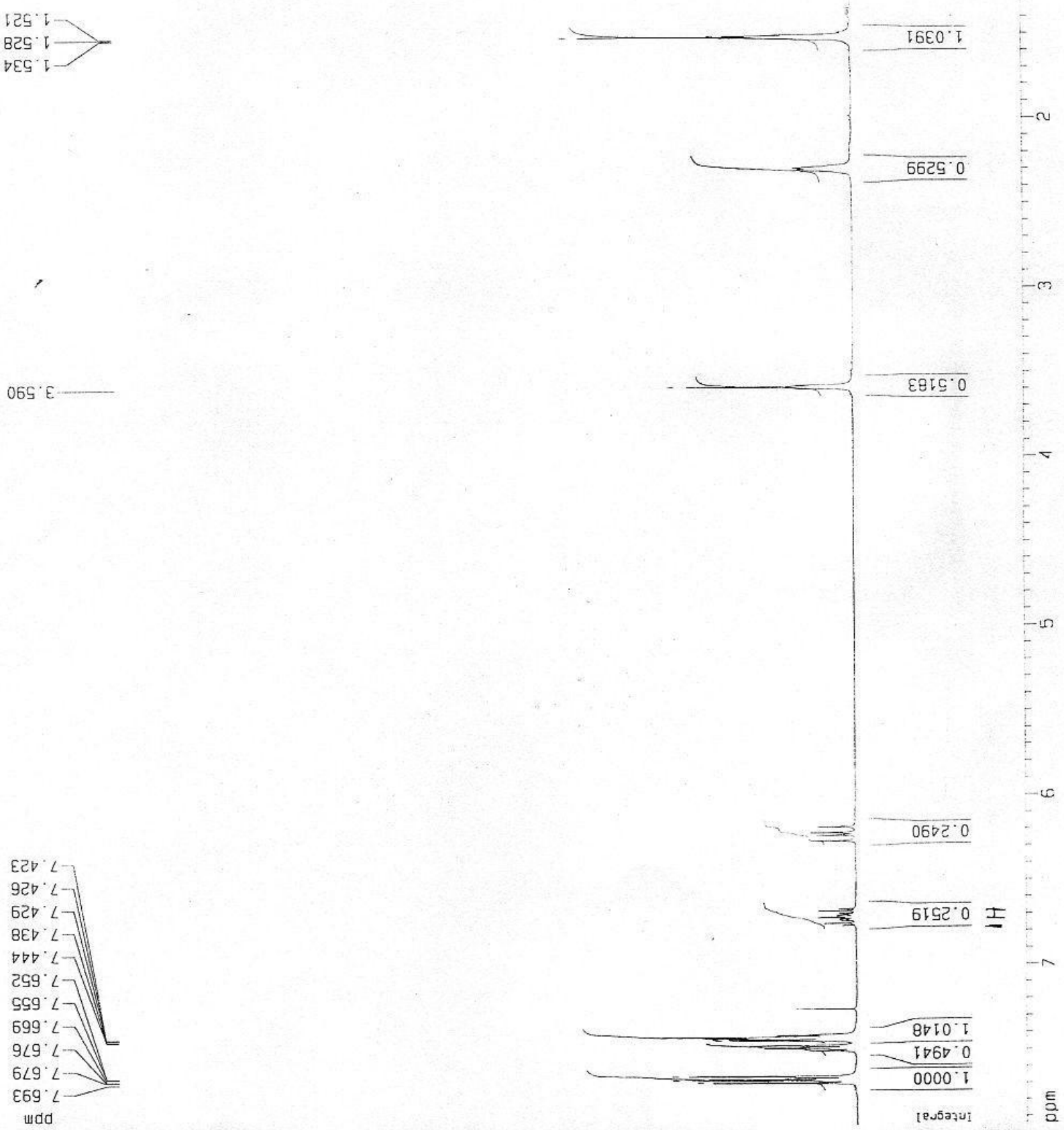

wdd 

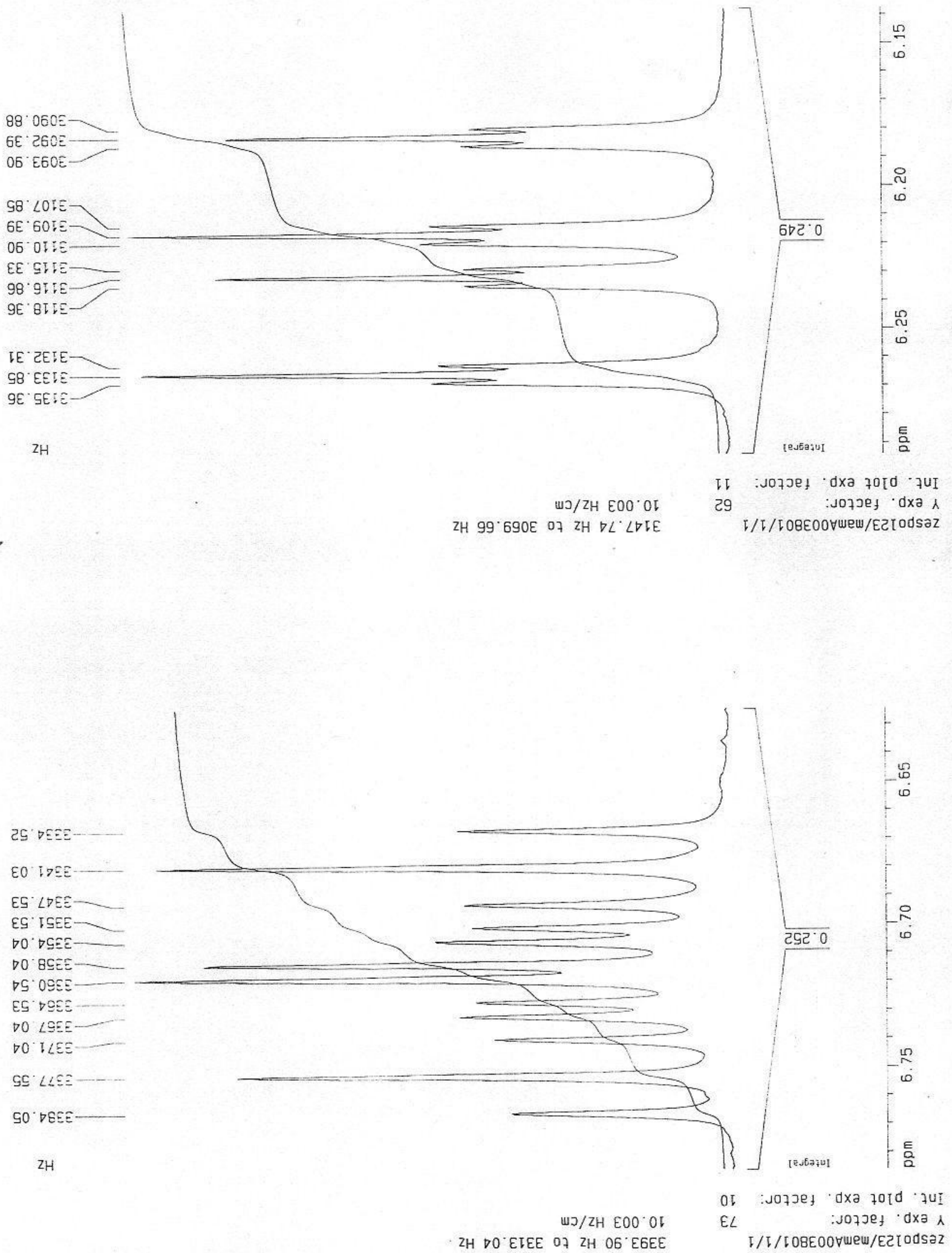


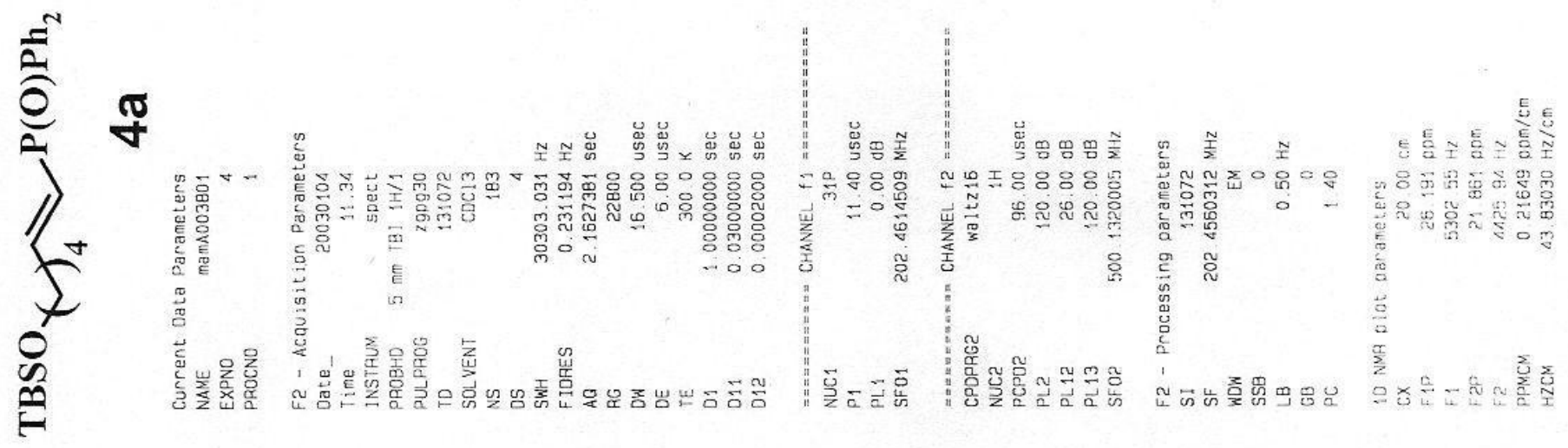

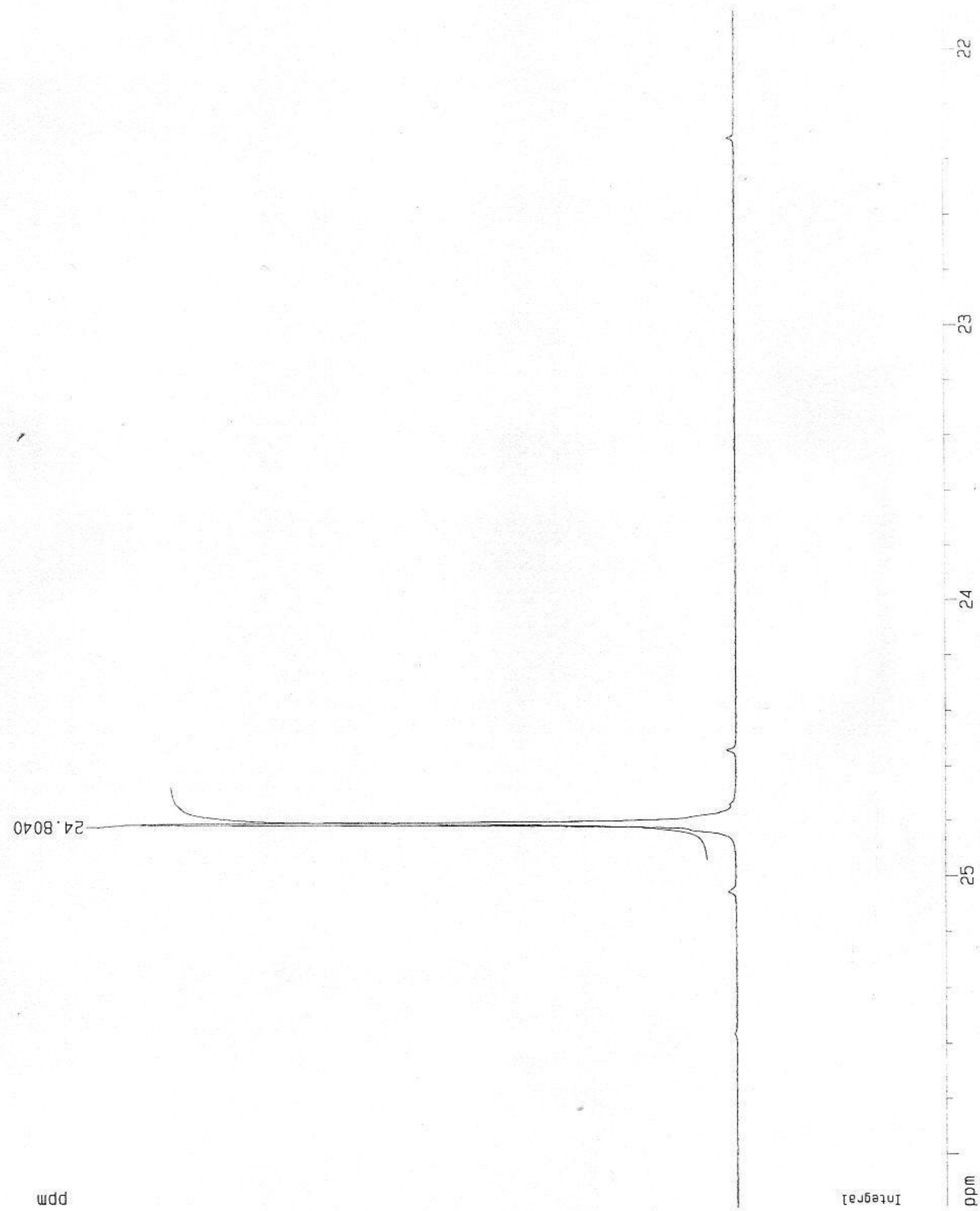


소소

令

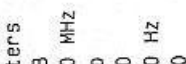

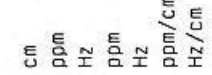

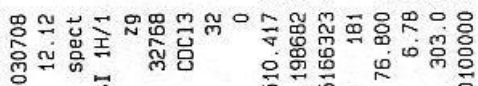

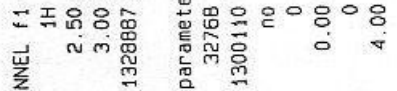

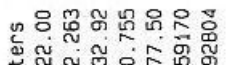

c

$000^{\circ}$

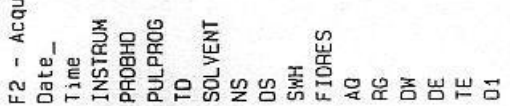

옹

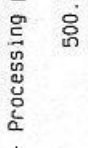

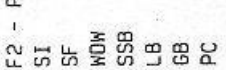

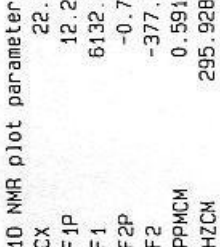

SII 2

96єट

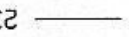

S92 $\mathrm{L}$

$E \angle D^{\circ} L$

IOL'L
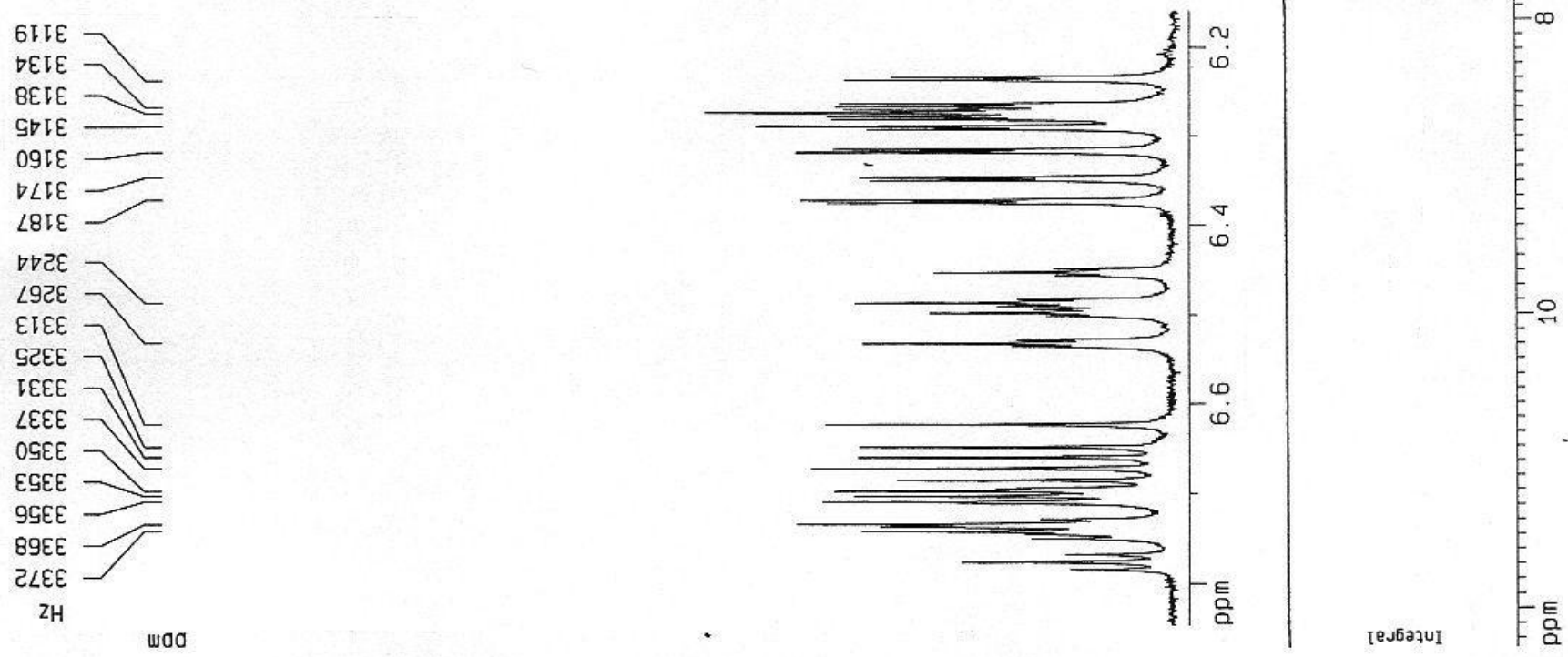


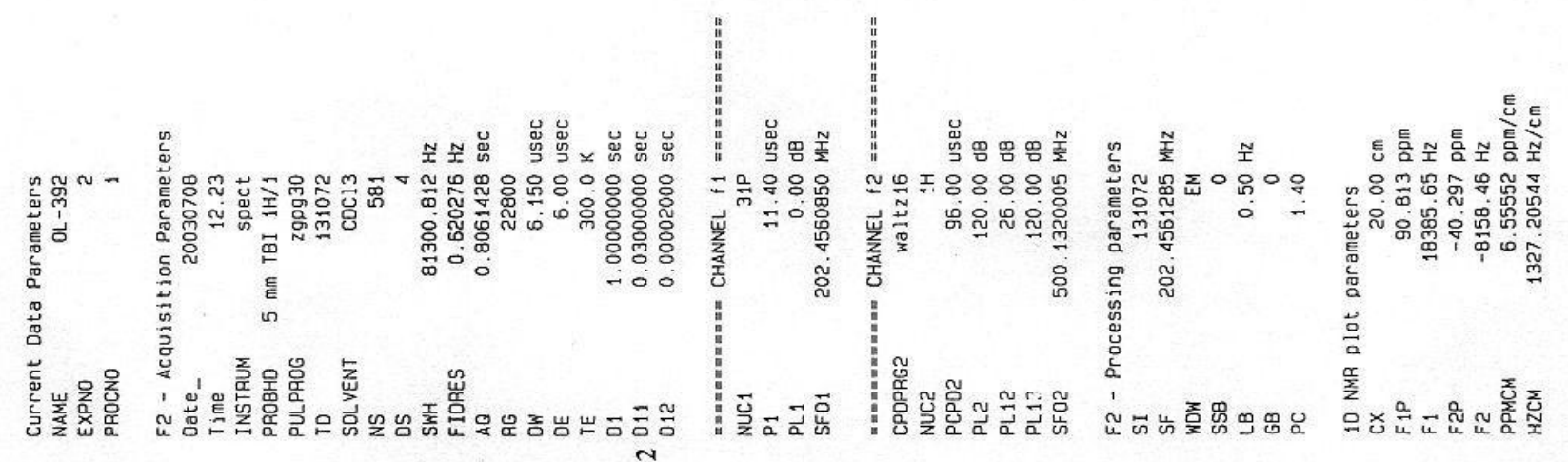

$6002 \cdot \nabla 2-$

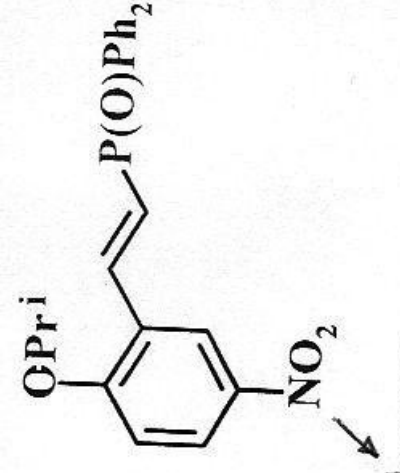

$0268 \cdot \nabla 2$

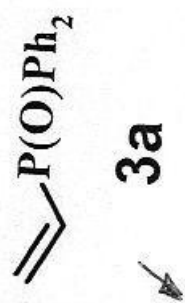

6076.0

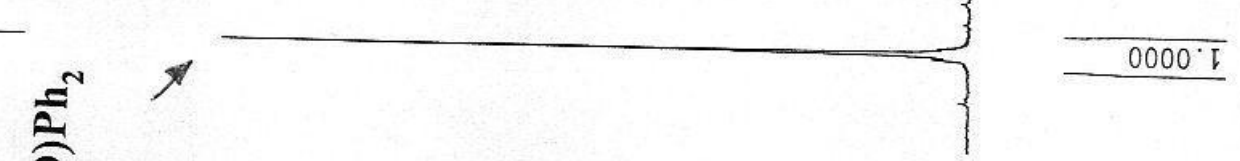

$6002 \cdot \nabla c-$

$0268^{\circ} \cdot \nabla 2-$ 


\section{RP-18 pomiar - Vial 1 Inj 1 - Channel 1}

Current Data Path: D:IHPLC-APLIKACJElOlegIDATAl0168 (oL-392)

Data Desc.: IFM CH1 2-D

Vial Number: 1 Inj Number: 1 Sample Name:

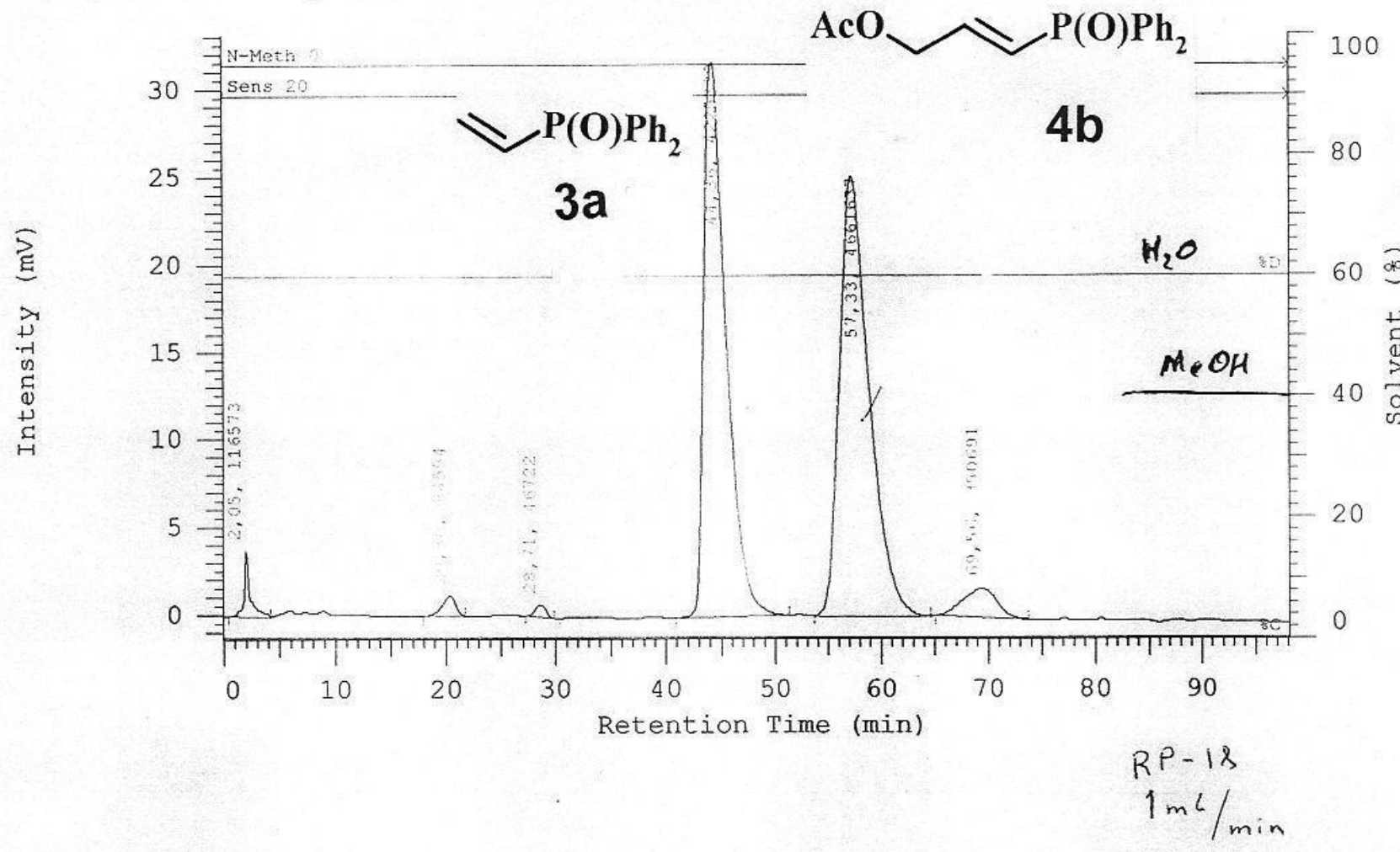

Li Chrospher 100 RP-is

$250 * 4 * 5 \mathrm{~mm}$

detector UV 


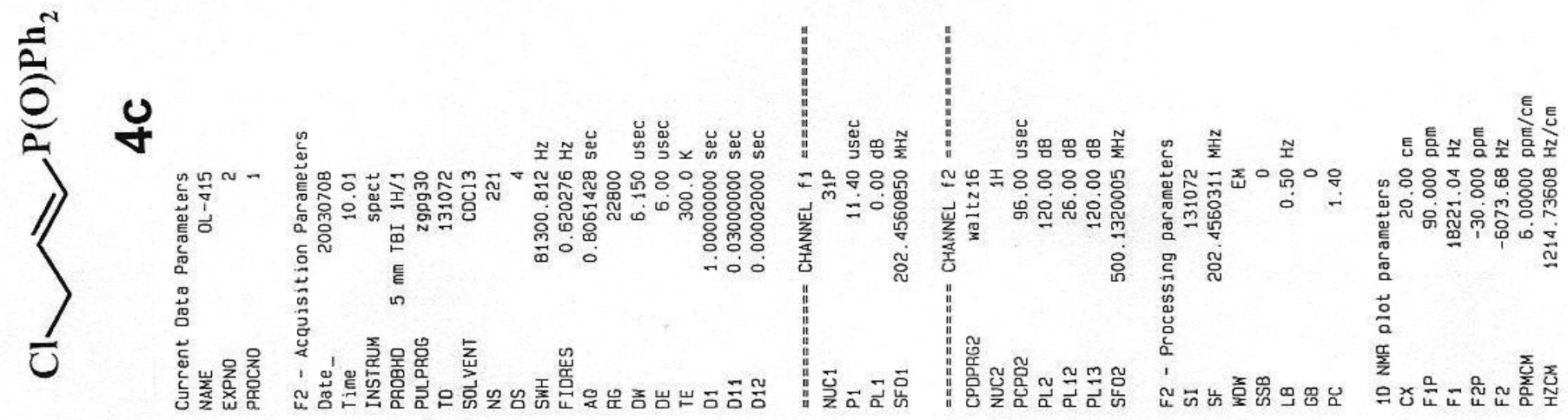

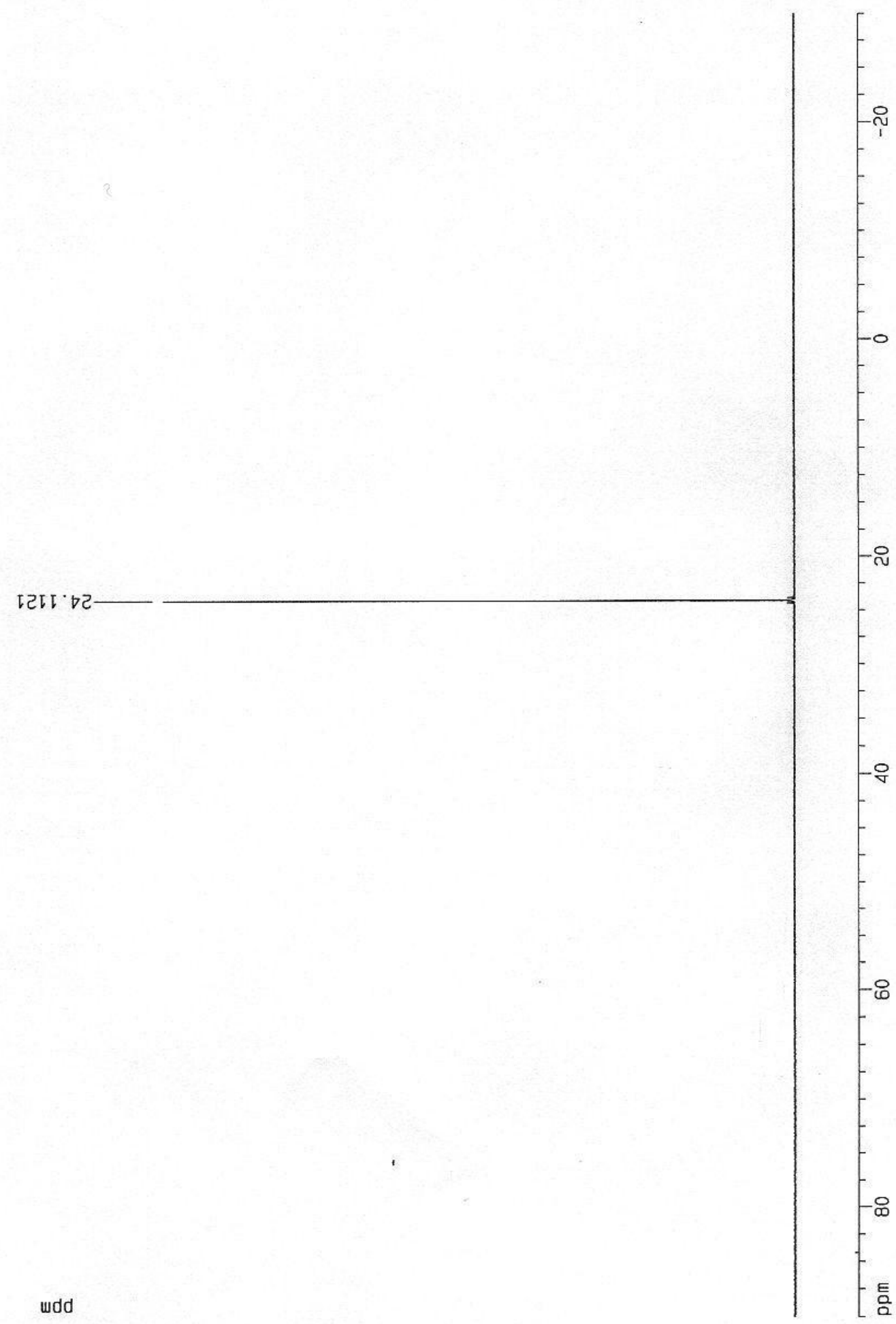




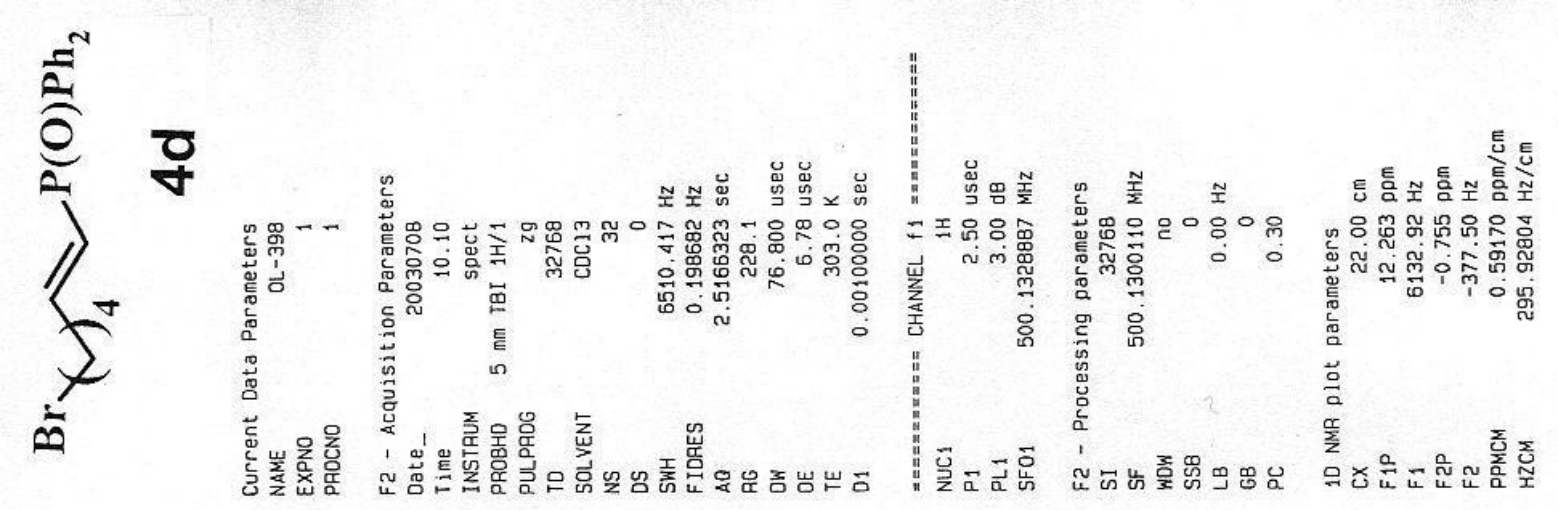

$\varepsilon 00^{\circ} 0$

$90 \nabla^{\circ} \varepsilon$

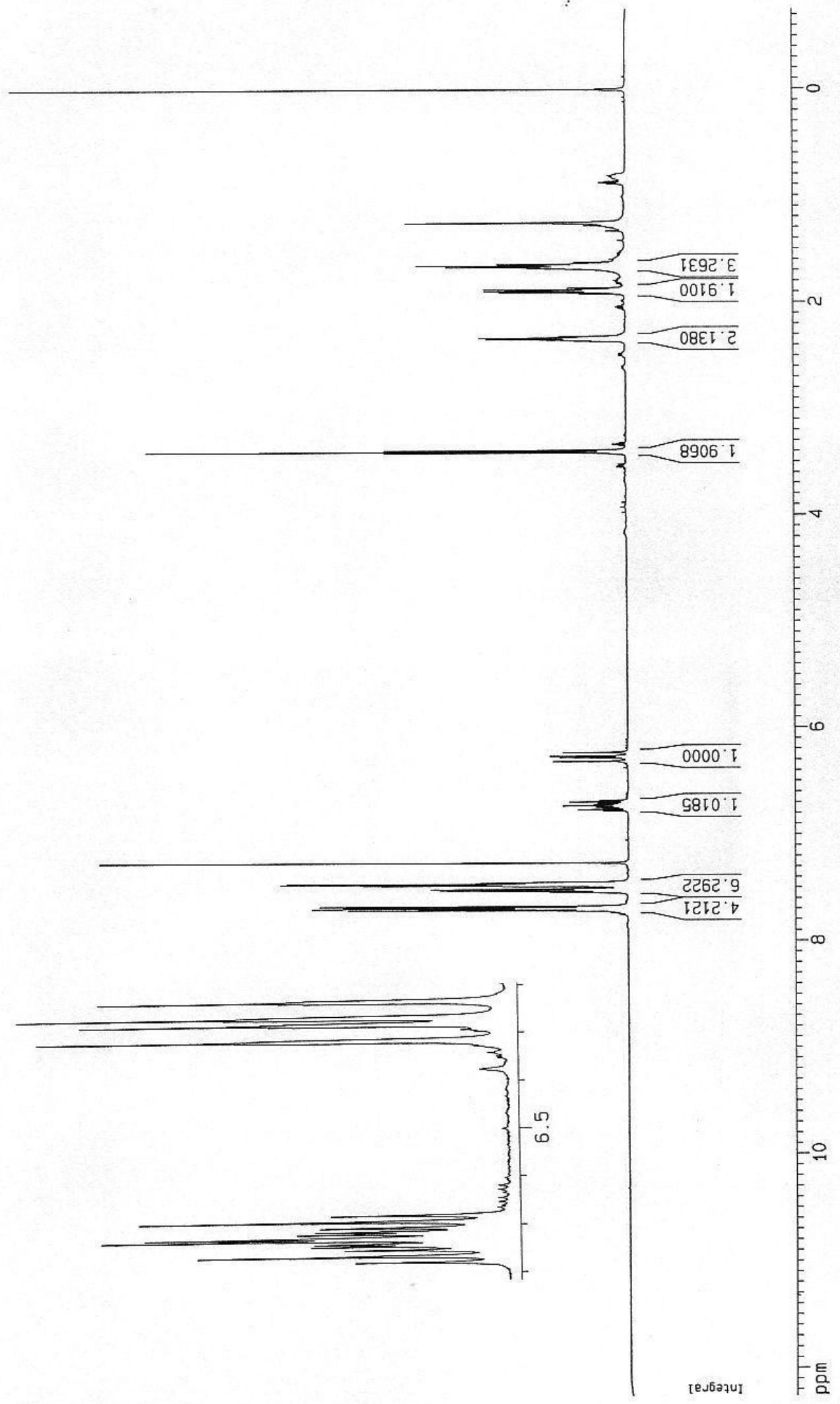



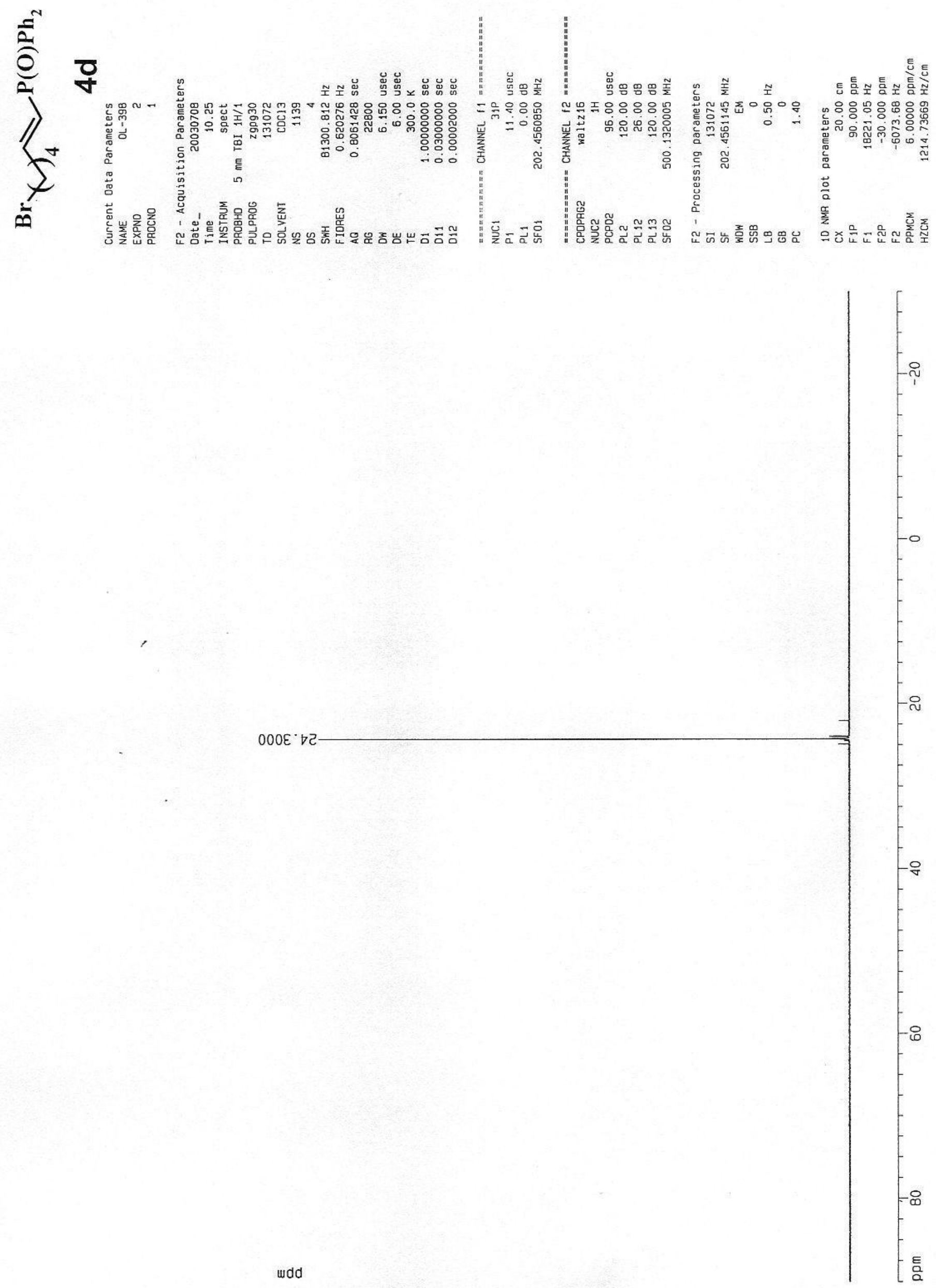
\&

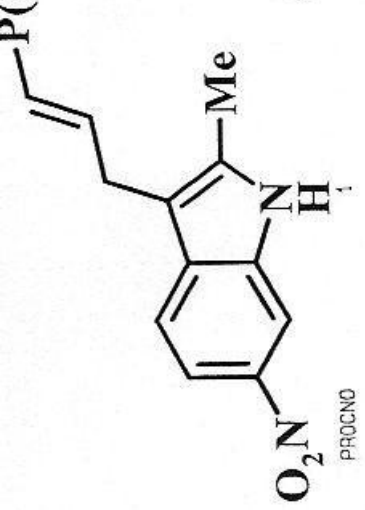

营

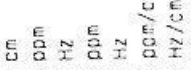

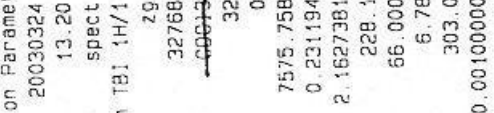

$E$
E
in

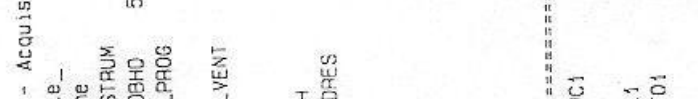

$\overbrace{}^{2}$

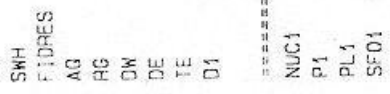

용

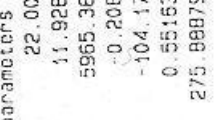

竞

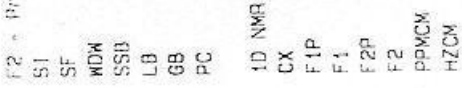

IBE

$8 \angle \nabla^{\circ} \mathrm{C}$

$980^{\circ} \mathrm{C}$

$680^{\circ} \mathrm{C}$

ट6० ट

982

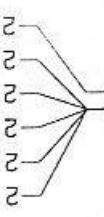

.

$69 \nabla^{\circ} \angle$

$\angle \angle \nabla^{\circ} \angle$
$\nabla \angle \nabla^{\circ} \angle$
$\nabla 80^{\circ} \angle$
$680^{\circ} \angle$
$\nabla 89^{\circ} \angle L$
$\angle 89^{\circ} \angle$
$109^{\circ} \angle$
$809^{\circ} \angle$
$019^{\circ} \angle$

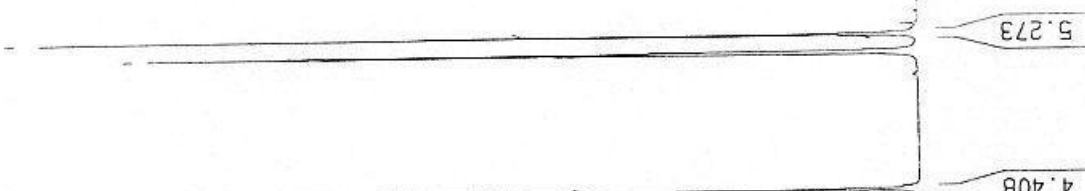

$=\frac{\operatorname{SgS} \varepsilon}{=}$

$\angle 90^{\circ} E$

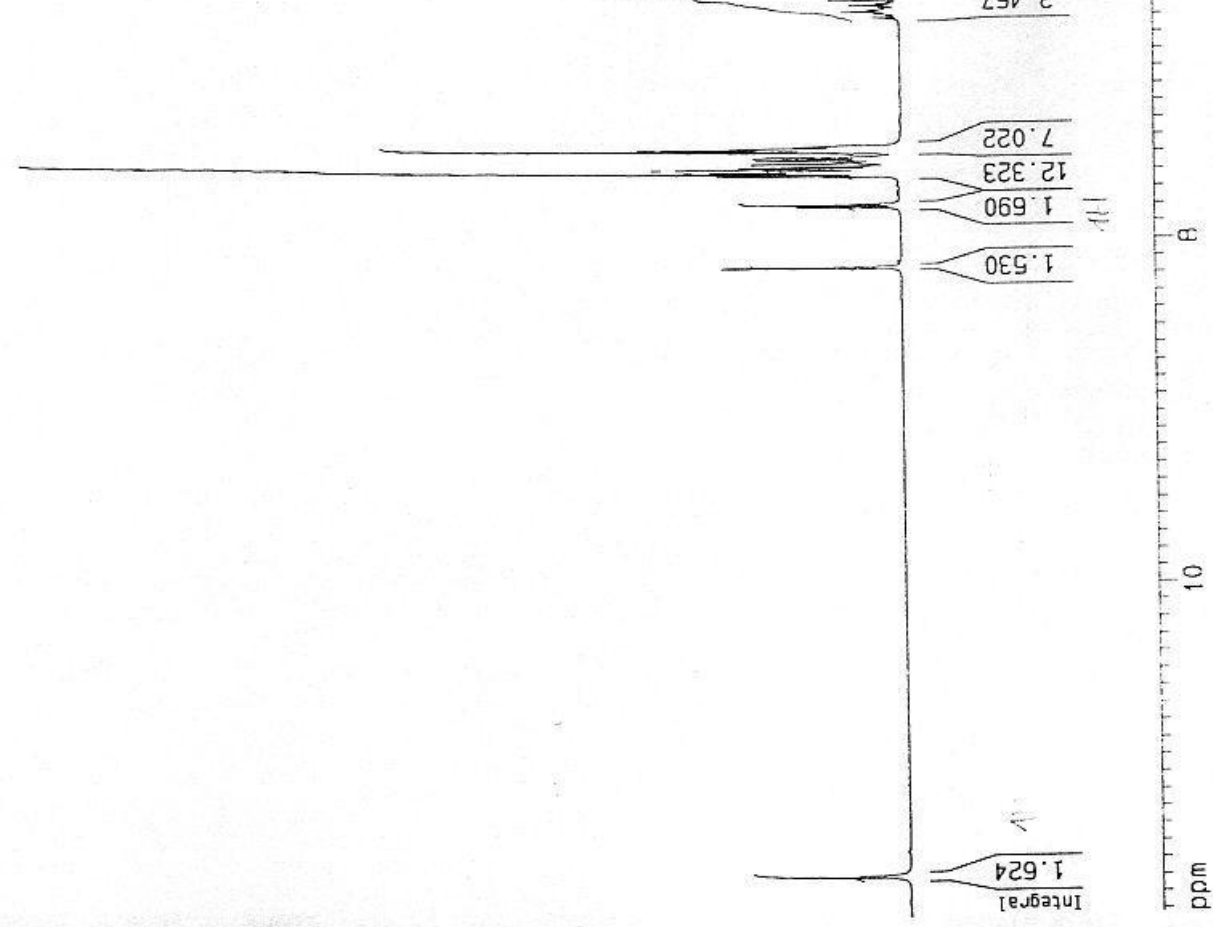

S- 16 


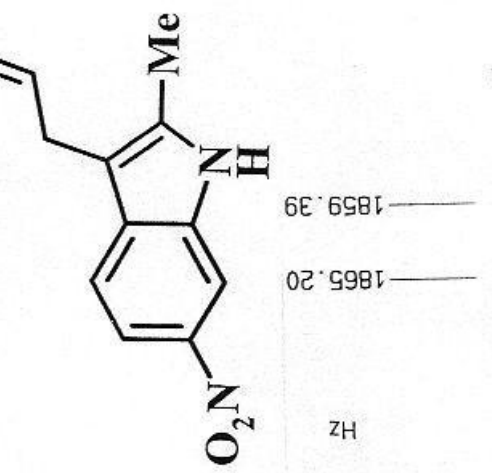

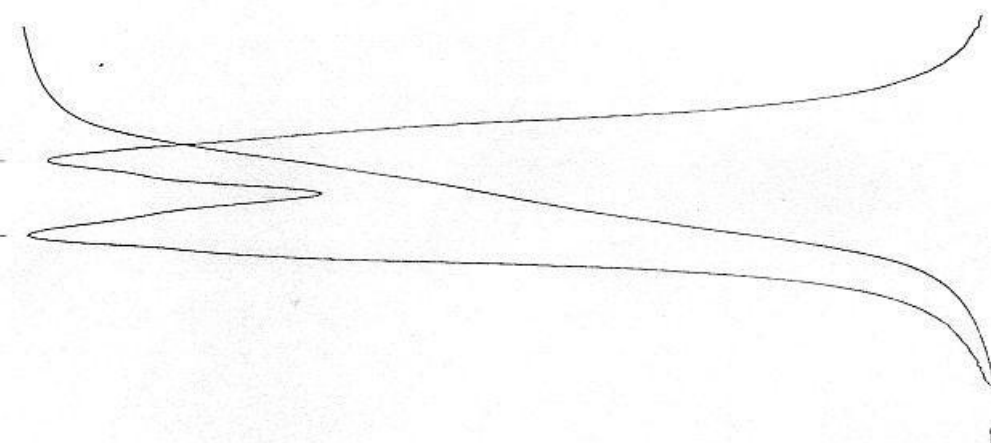

wว/ZH $205^{\circ} \angle$ $\mathrm{ZH} 09^{\circ} 978 \mathrm{I}$ of $\mathrm{ZH}$ 6I. $8 \angle 8 \mathrm{I}$

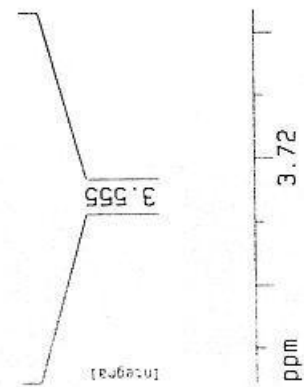

:.ло7jet dxa zold zui :.107כ5ef dxa $A$ ఒ/I/OOBGOV/EZTOdsaz
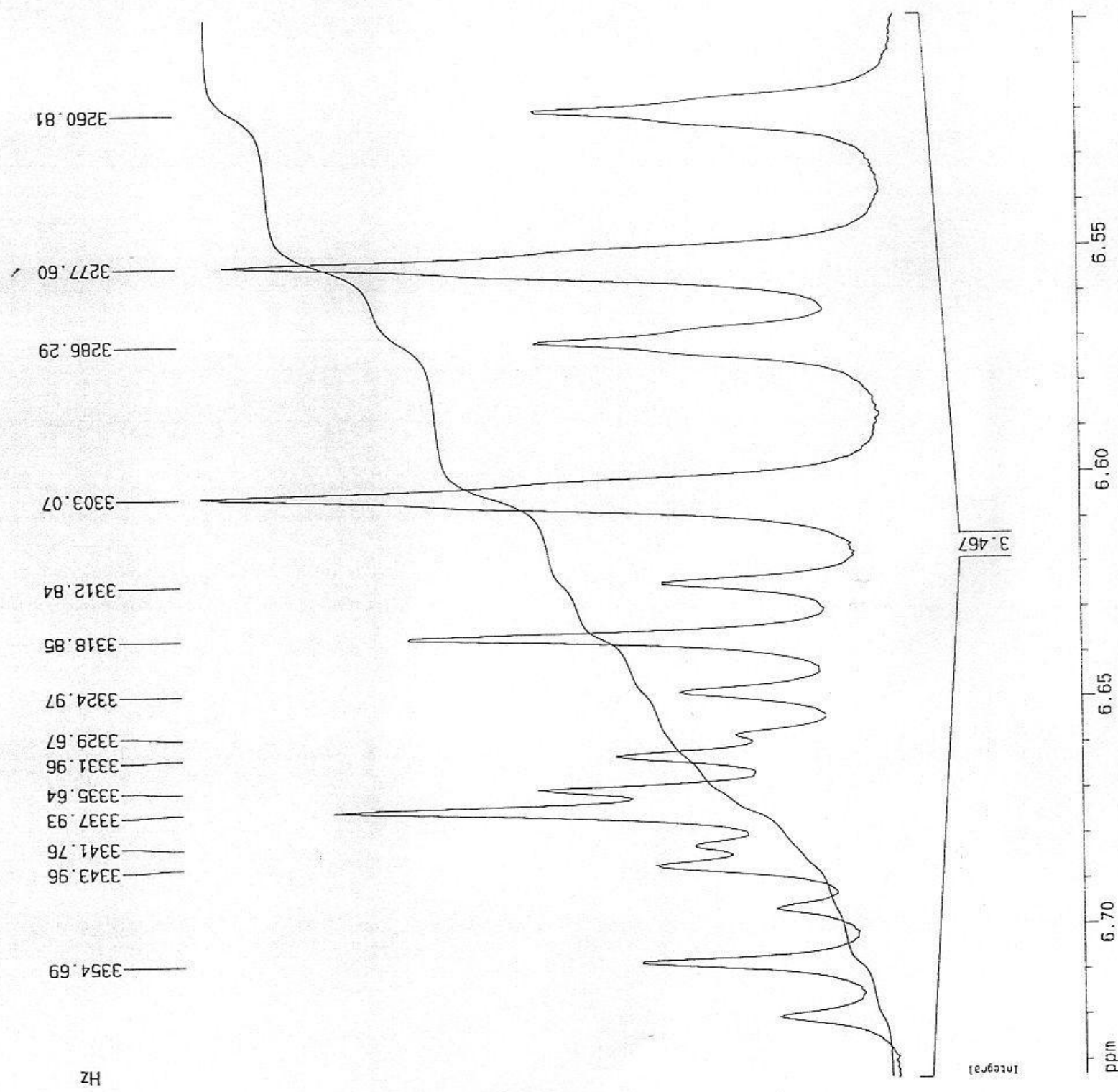

шว/ZН $209^{\circ} \angle$ $\nabla$ ZH 60. OSटE OF ZH $\nabla 9^{\circ}$ L9EE 

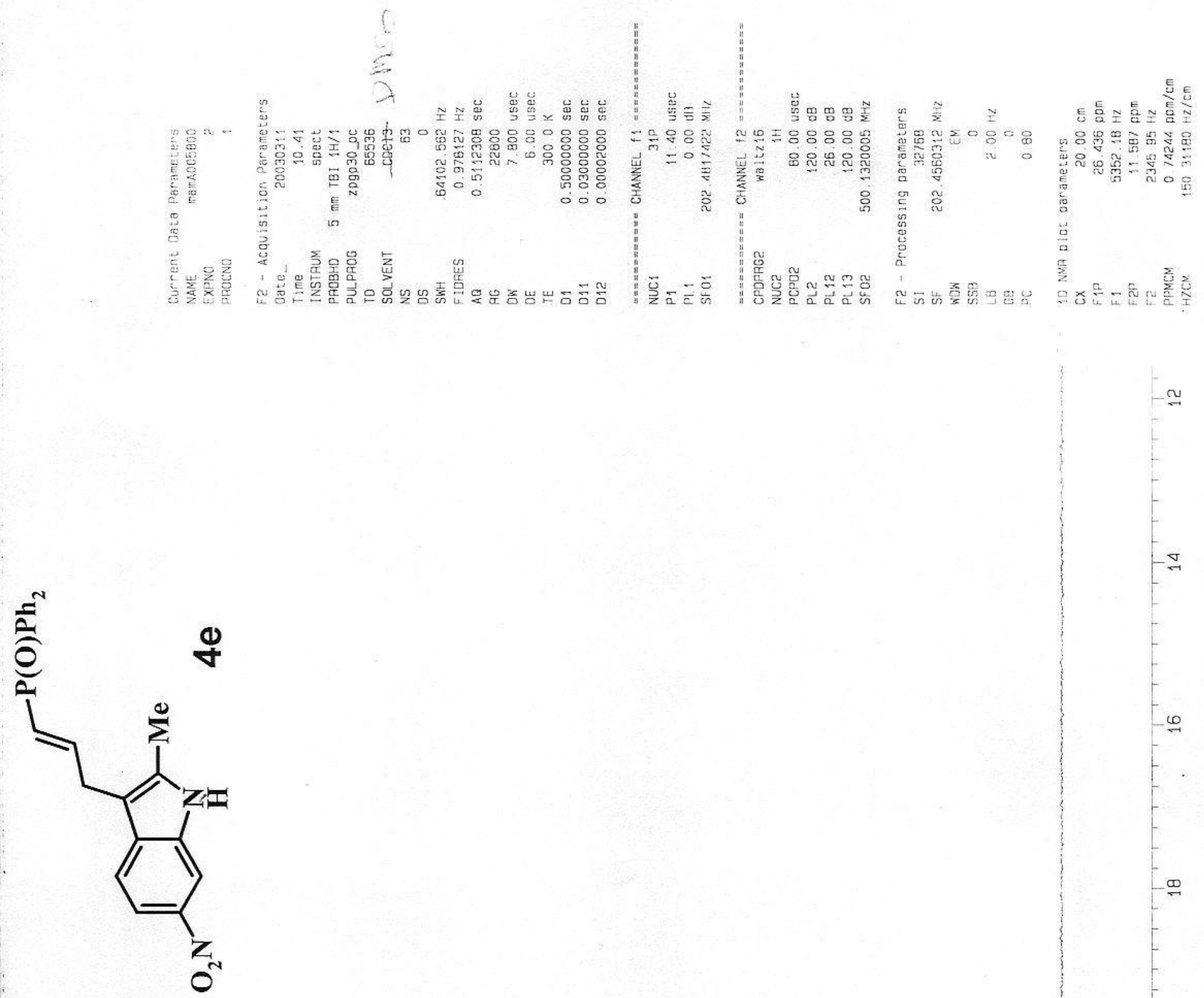

$\nabla G L D D^{\circ}$ 


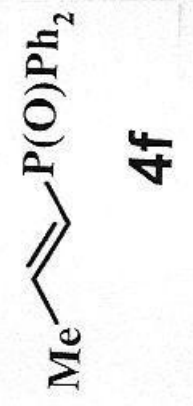

Ingl

营

조소욨

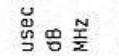

좊 존

$\begin{array}{lll} & \\ 0 & \end{array}$

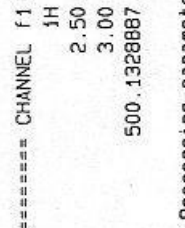

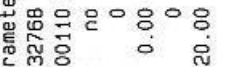

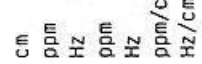

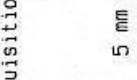

蛋

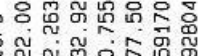

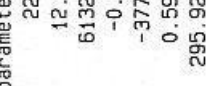

。

음

等

${ }_{\nabla 66}^{086}: \longrightarrow$
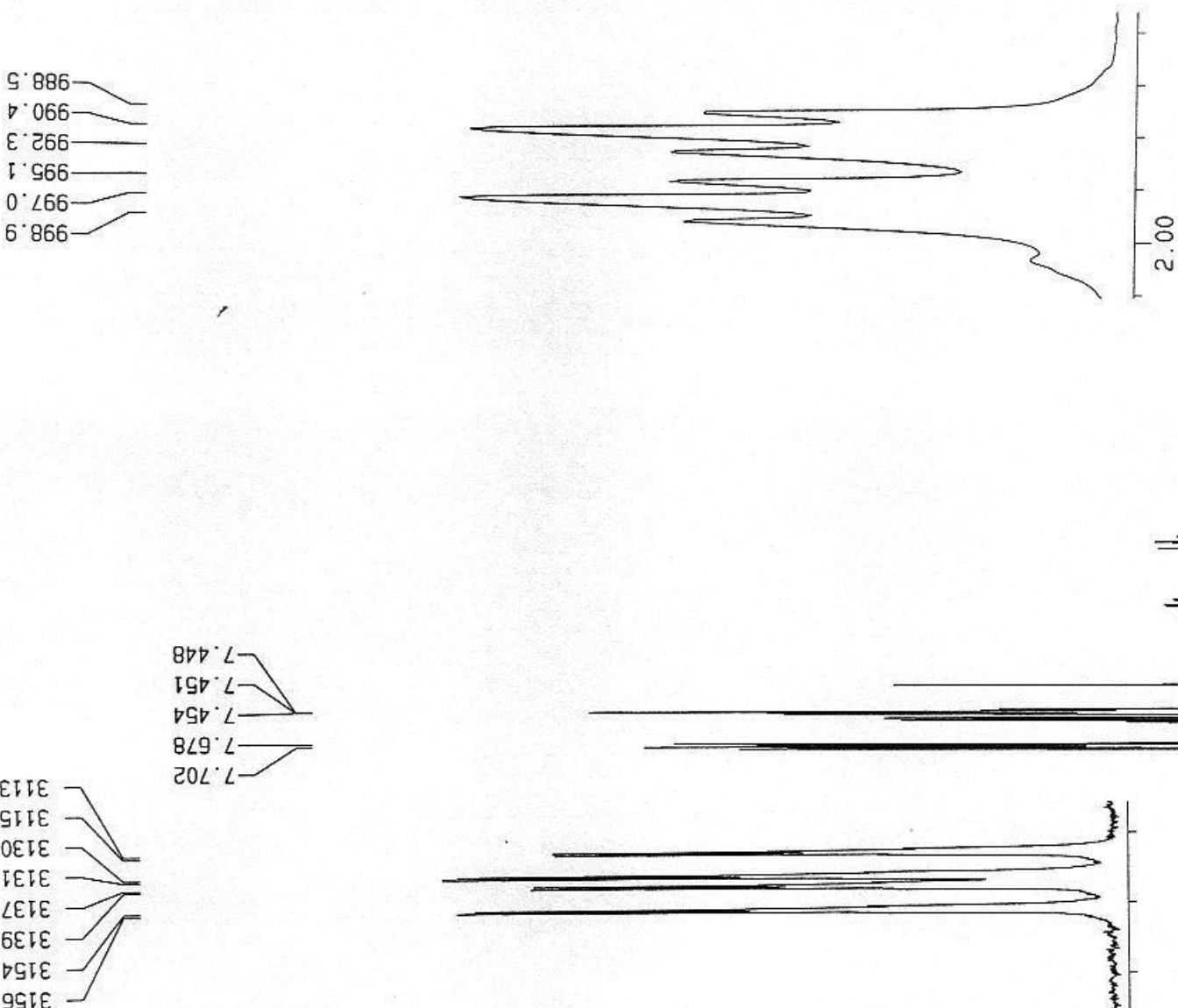

प9

$8 \angle 9^{\circ} \angle$ $20 L^{\circ} L$

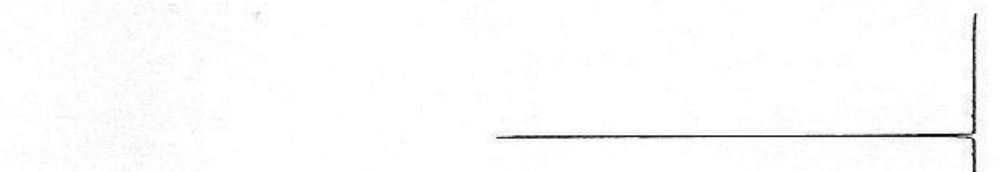

. 

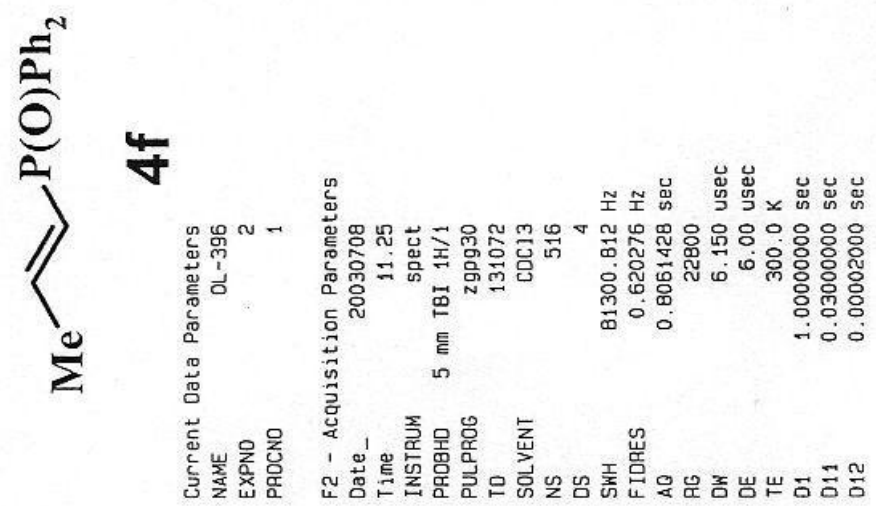

茛品等

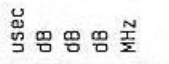

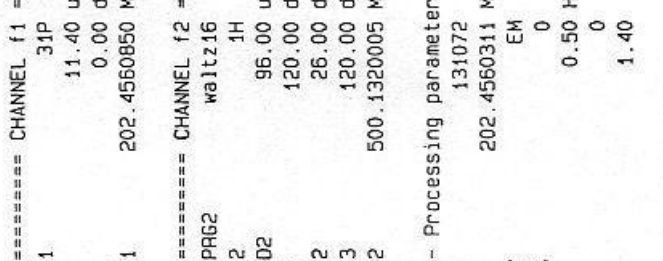

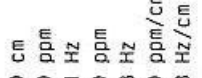

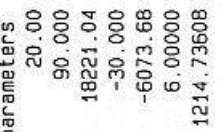

粱

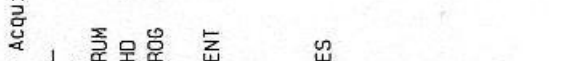

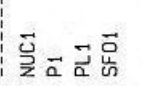

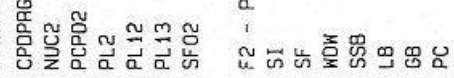
亭

峷

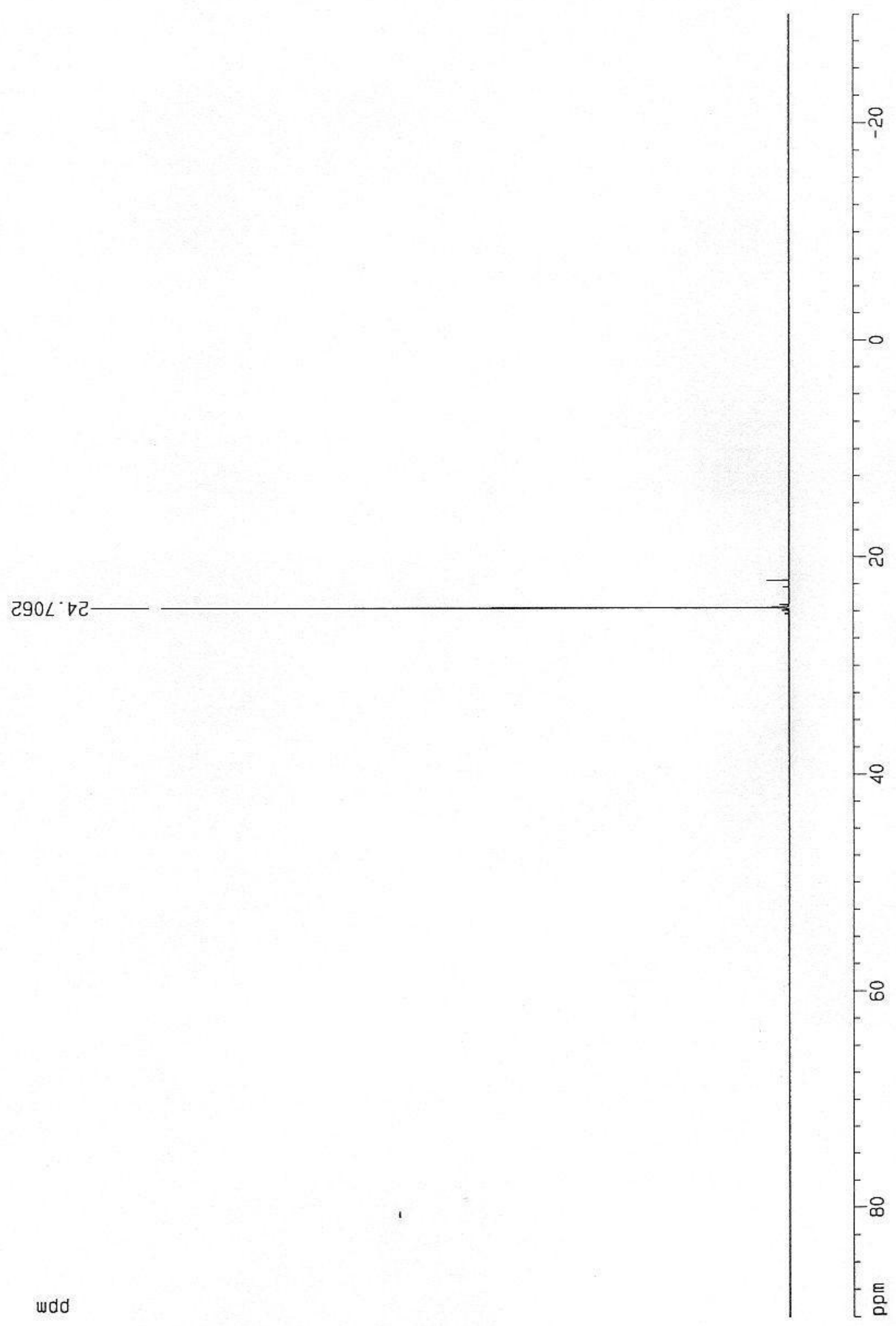



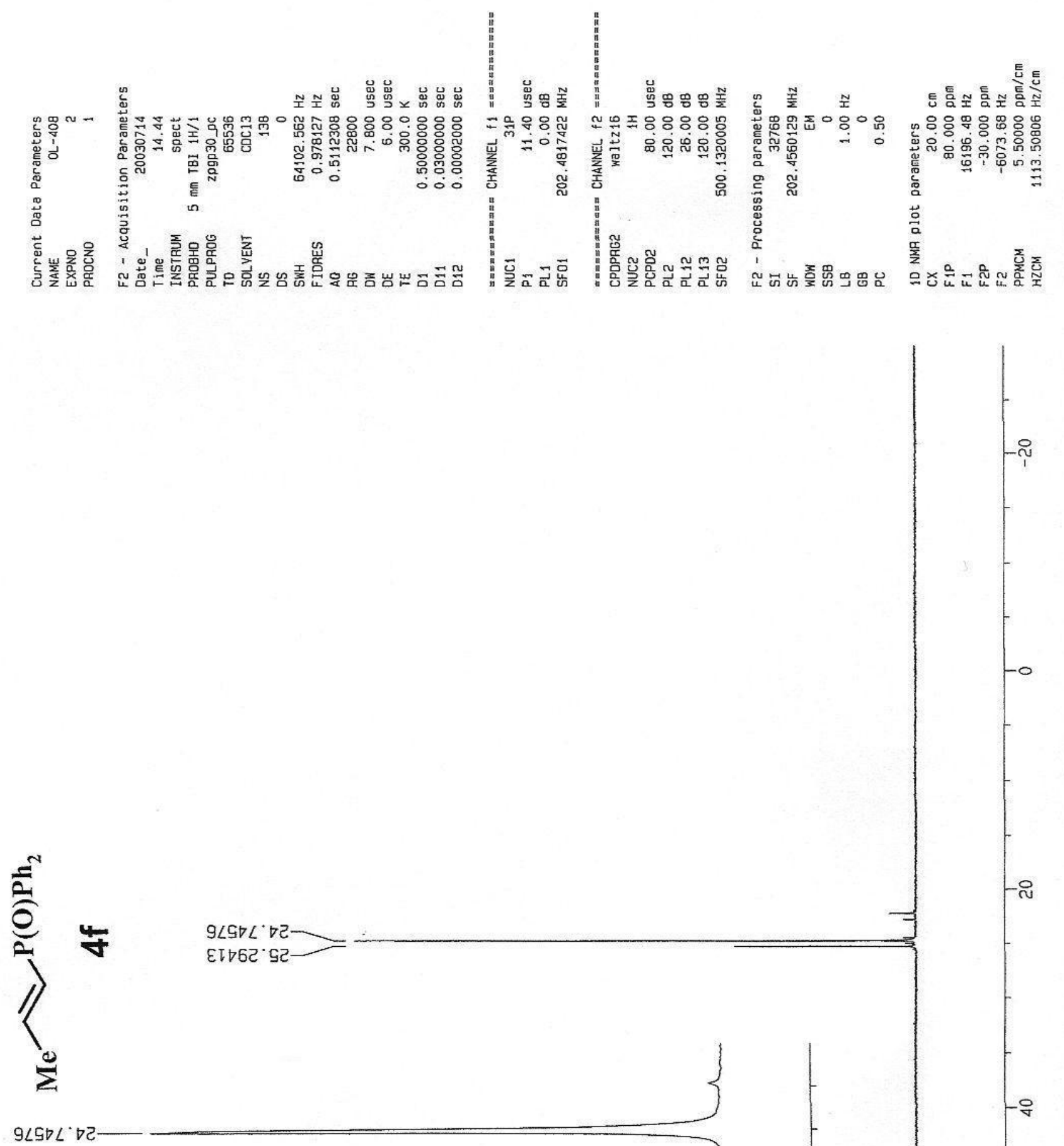

ยเจธट 92

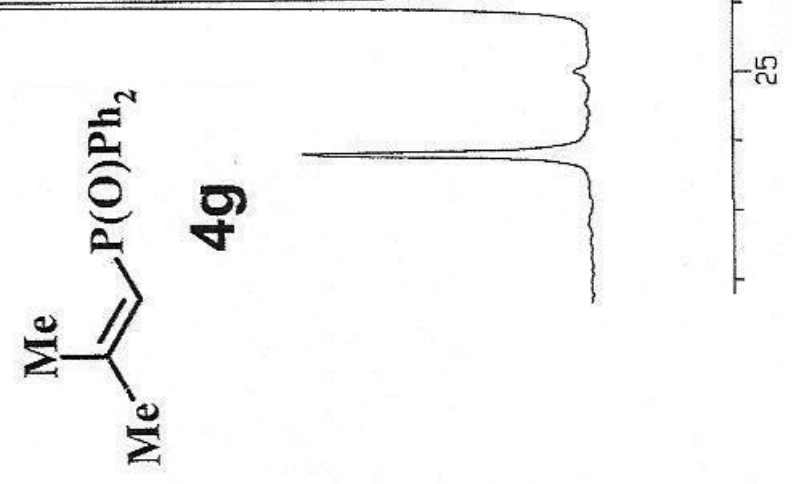




\section{RP-18 pomiar - Vial 1 Inj 1 - Channel 1}

Current Data Path: D:LHPLC-APLIKACJElOleg\DATAl0181

Data Desc.: IFM CH1 2-D

Vial Number: 1 Inj Number: 1 Sample Name:

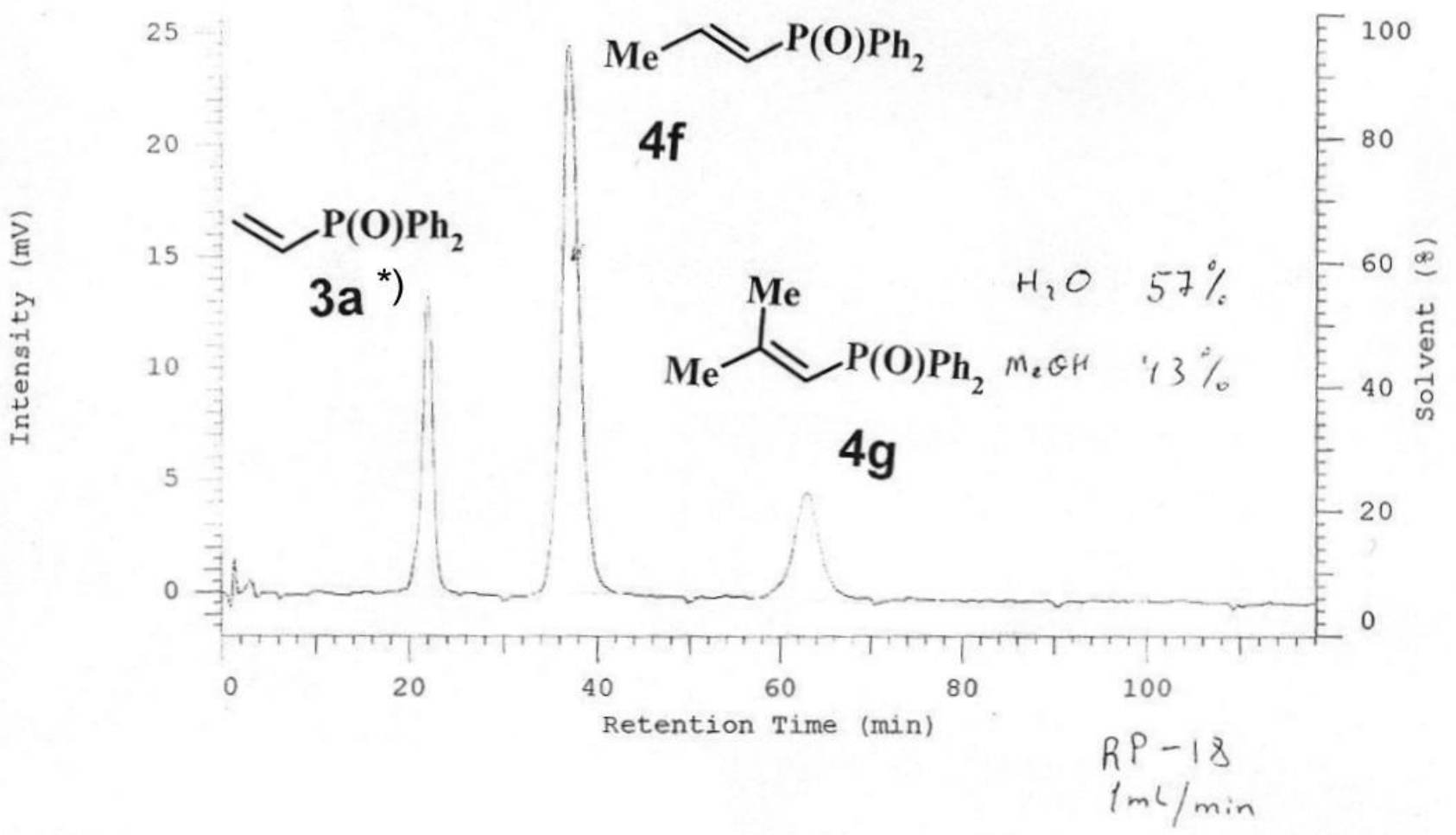

*) added as internal standard

LiChrospher 100 RP-18 $250 * 4 * 5 \mathrm{~mm}$
detector UV 
$\angle 619.1-$

EDEL '

LOSL '

9อ9B. [-

$B E \angle E^{\circ} E$

¿ $\angle B E^{\circ} E$

$D 00 D \cdot E-$

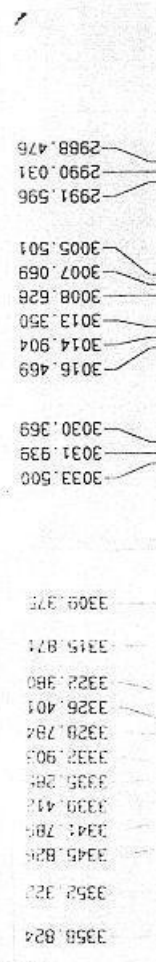

$1 / 52^{\circ} \mathrm{L} \ldots$

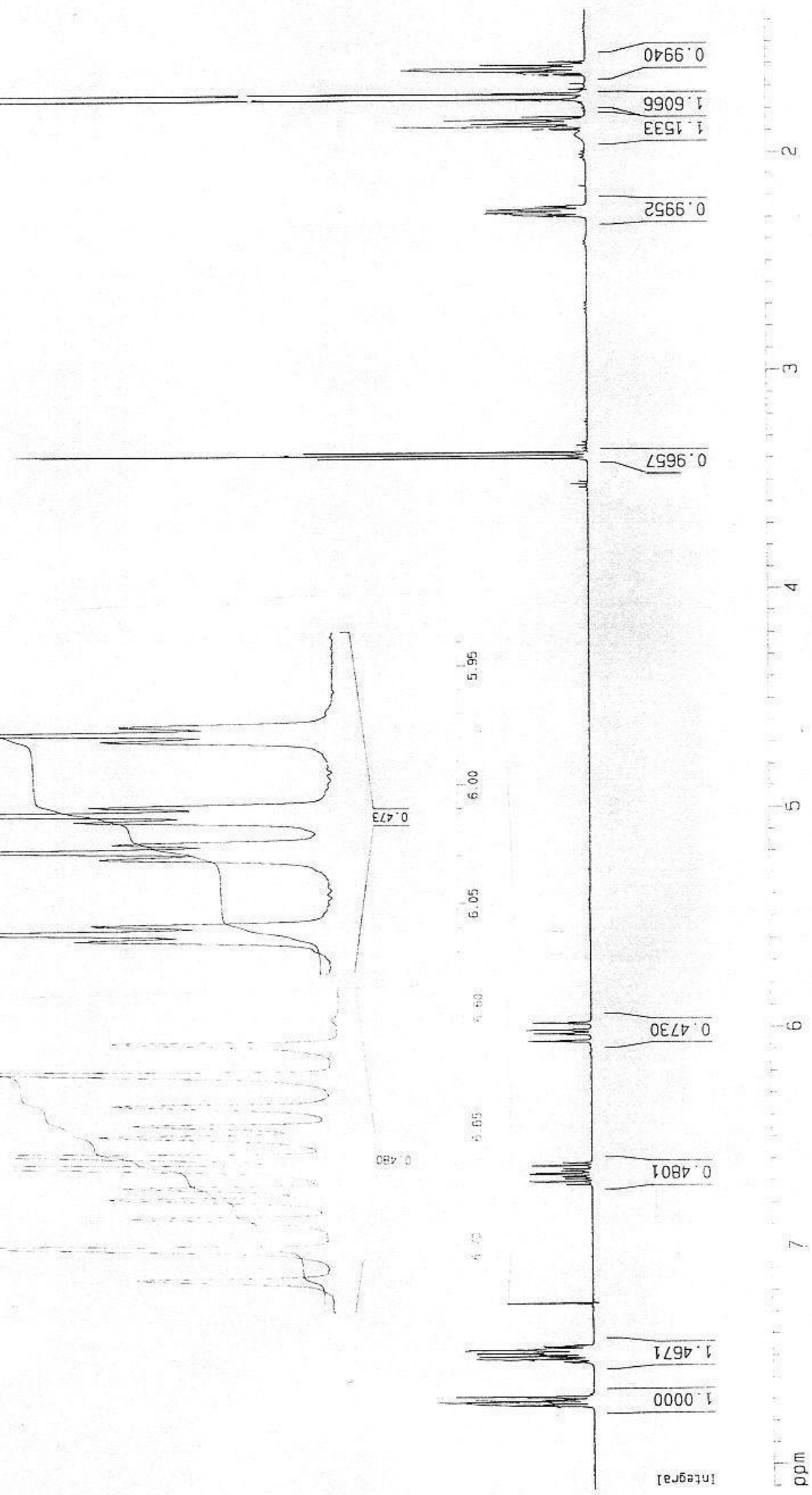



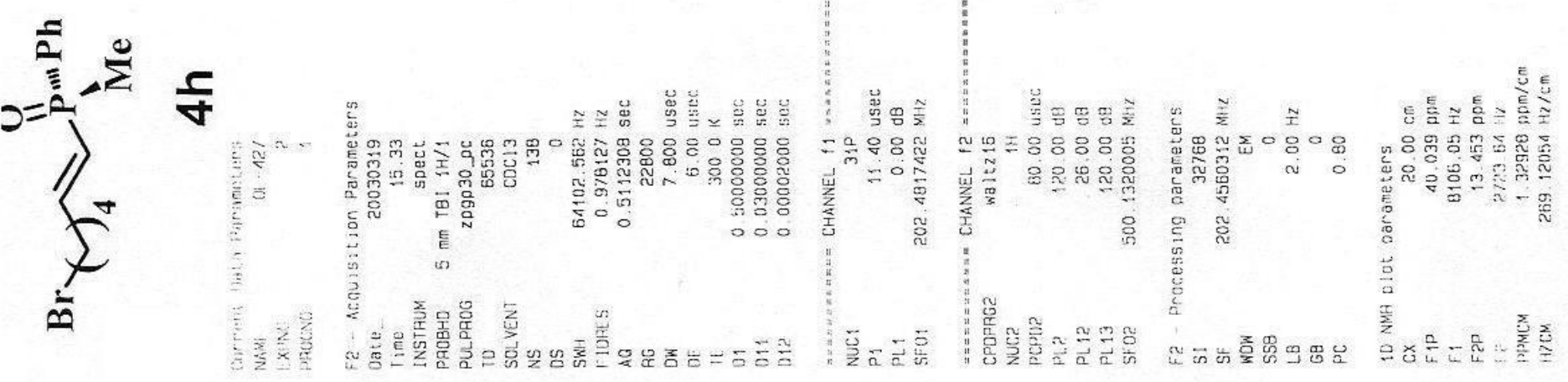

GGDGE $\angle 2-$ 


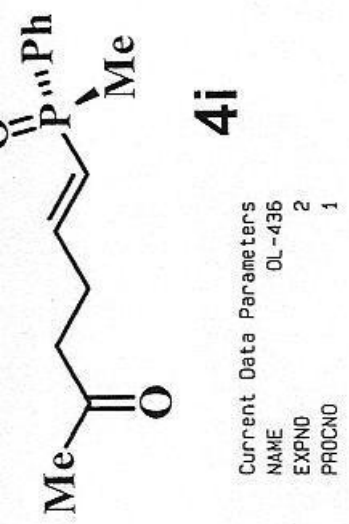

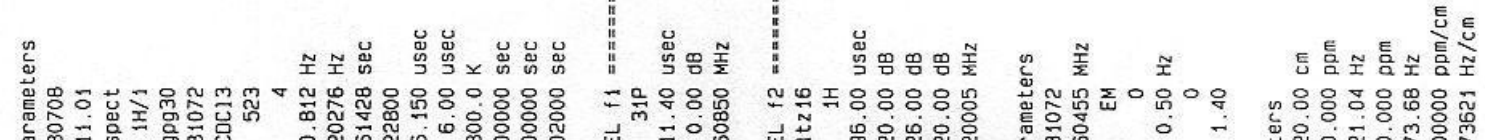

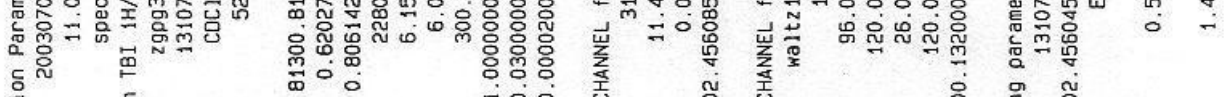

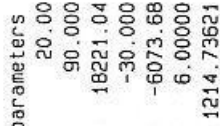

营

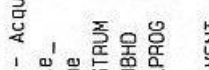

宧

:

드 ๙ั

$\stackrel{\circ}{\circ}$

变

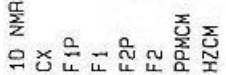

6टाЕ 


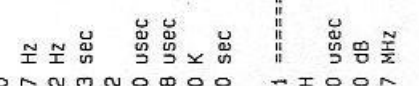

is

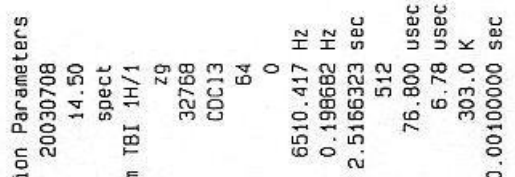

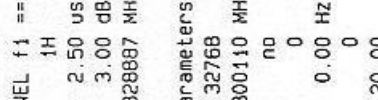

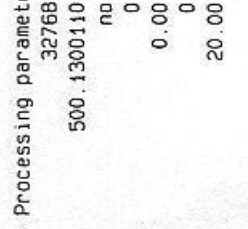

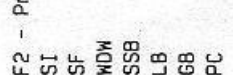

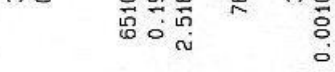

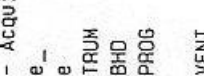

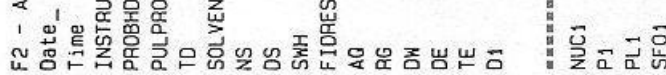

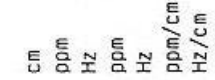

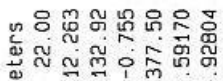

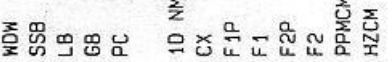

$500^{\circ}$

$\angle O O^{\circ} \cdot \mathrm{L}=$

$609^{\circ}$

乌टg. $\varepsilon>$

EIBI

6เ81

$\angle \varepsilon 9^{\circ} \varepsilon$
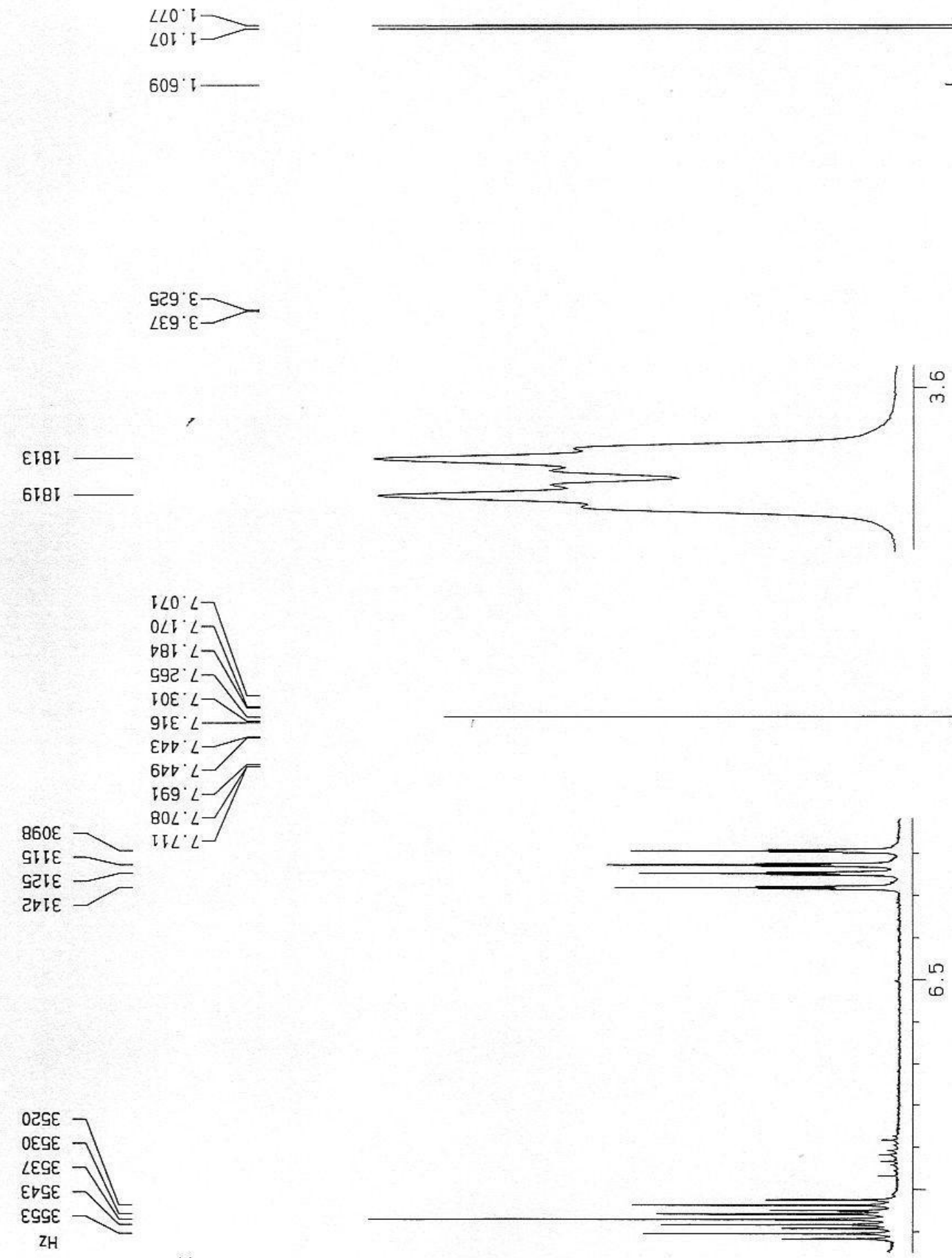

$=0$ 


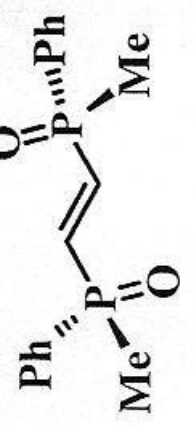

10

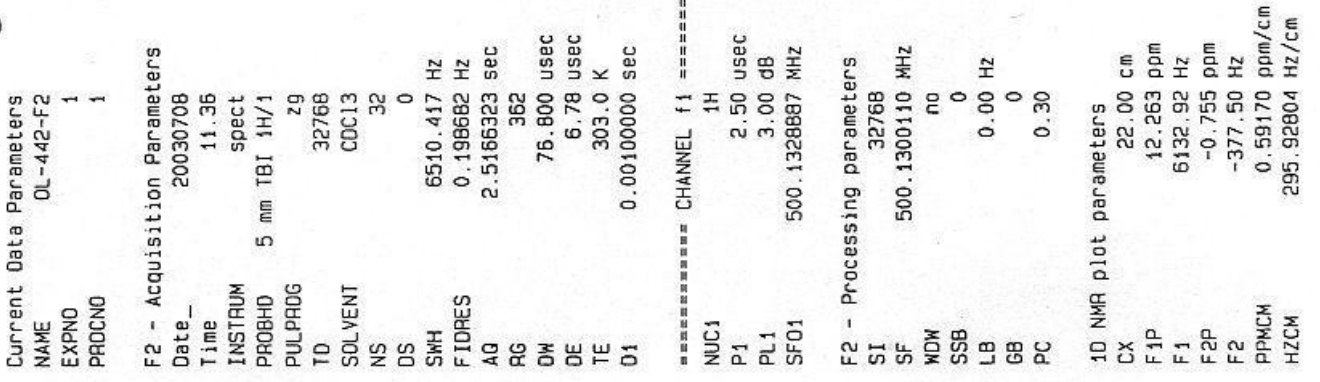

$100 \%$

$\angle$ L L6 -

$\varepsilon \cdot \nabla 26$

8. $0 \varepsilon 6$

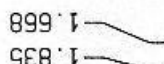

I98.

\section{ontaly}
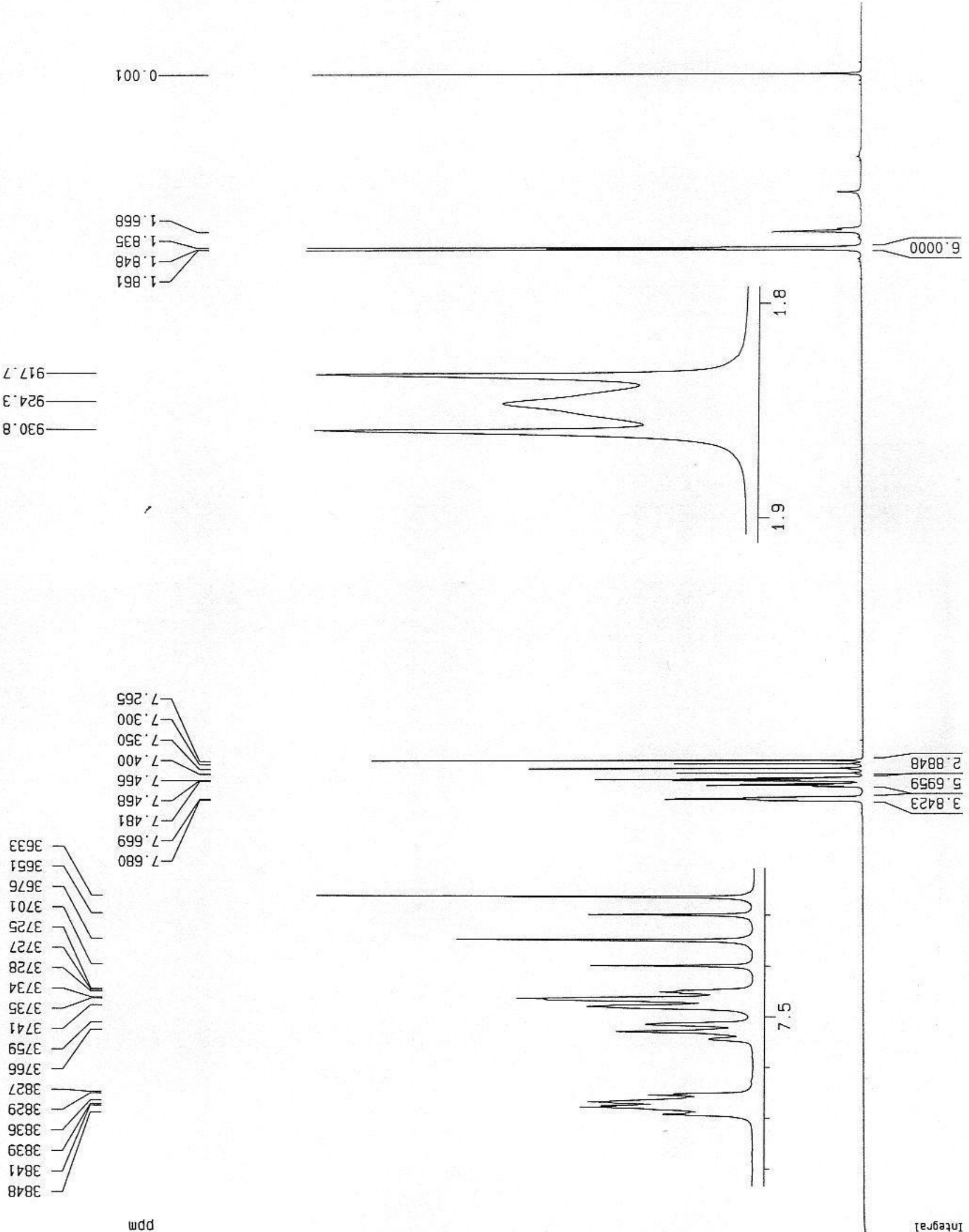


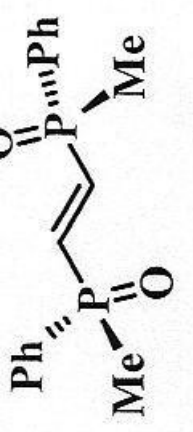

10

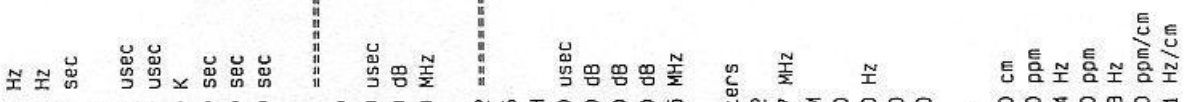

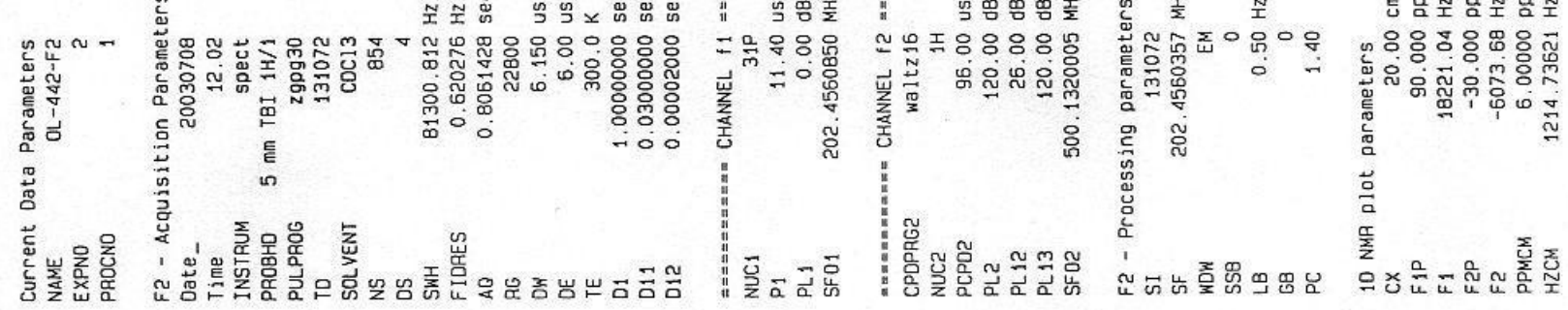


